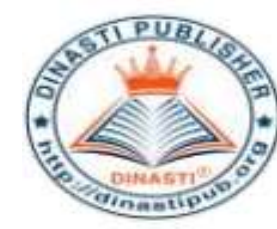

\title{
COMPARISON OF EFFECTIVENESS INVENTORY CONTROL OF EOQ METHOD WITH COMPANY METHOD IN STEEL MATERIAL FABRICATION KRAKATAU POSCO
}

\section{Gunawan Adam ${ }^{1}$, Tukhas Shilul Imaroh ${ }^{2}$}

${ }^{1)}$ Mercu Buana University, Jakarta, Indonesia

${ }^{2)}$ Mercu Buana University, Jakarta, Indonesia

\begin{tabular}{|c|c|}
\hline $\begin{array}{l}\text { ARTICLE INFORMATION } \\
\text { Received: } 20 \text { December } 2019 \\
\text { Revised: 24 December } 2019 \\
\text { Issued: } 25 \text { December } 2019 \\
\text { Corresponding author: first author } \\
\text { E-mail: } \\
\text { gunawanadam08@ gmail.com } \\
\text { tukhas.shilul@mercubuana.ac.id } \\
\text { DOI:10.31933/DIJMESS }\end{array}$ & $\begin{array}{l}\text { Abstract: PT. Krakatau Posco, is the largest steel } \\
\text { manufacturing company in Southeast Asia that } \\
\text { produces slabs and plates. This study aims to compare } \\
\text { the effectiveness of controlling inventory of steel } \\
\text { material fabrication workshop with the EOQ method } \\
\text { and compared with the current method of the company } \\
\text { in an effort to prevent the unavailability of such steel } \\
\text { material fabrication. Inventory control using the EOQ } \\
\text { method is expected to provide solutions for inventory } \\
\text { control, and optimal ordering in order to obtain } \\
\text { savings for inventory costs (Total Inventory Cost) at } \\
\text { PT. Krakatau Posco. The method used is an interview } \\
\text { and take the necessary data, then conduct an initial } \\
\text { analysis using the ABC method to clarify the data that } \\
\text { has been carried out at PT. Krakatau Posco, controls } \\
\text { inventory using EOQ, determines safety stock, ROP } \\
\text { and maximum inventory. By controlling the ordering } \\
\text { and inventory also expected to provide significant } \\
\text { savings for the company, by reducing inventory costs } \\
\text { (Total Inventory Cost). The conclusion of this research } \\
\text { is the EOQ method is the right method for controlling } \\
\text { steel raw material fabrication to meet the production } \\
\text { and repair processes at PT. Krakatau Posco and with } \\
\text { this method the results of total inventory costs are also } \\
\text { more efficient. } \\
\text { Keywords: Inventory control, EOQ, ROP, safety } \\
\text { stock, total inventory cost }\end{array}$ \\
\hline
\end{tabular}

\section{INTRODUCTION}

Krakatau Posco is a company engaged in manufacturing that produces steel Slabs and Plates. As an integrated steel plant that has the first Blast Furnace technology in Indonesia, this Posco krakatau has 5 plants and has an integrated steel material system which means that all are integrated with one another. As well as having a centralized steel material fabrication 
workshop, where any damage to machine components or facility components is repaired here. This steel material fabrication stock consists of various types of materials such as angles, channels, expanded metal, flat bars, H-beams, I-beams, pipes and round bars.

All this time, material fabrication inventory control only estimates the availability of raw materials available to meet production needs such as, how many have to order, when to order, how much maximum inventory should be stored in the warehouse, how much inventory must be in the warehouse (safety stock ) so that there is no shortage or excess, as listed in table 1.1 excerpt of Steel Material stock 2018.

\section{Tabel 1. Steel Material Stock List Krakatau Posco 2018}

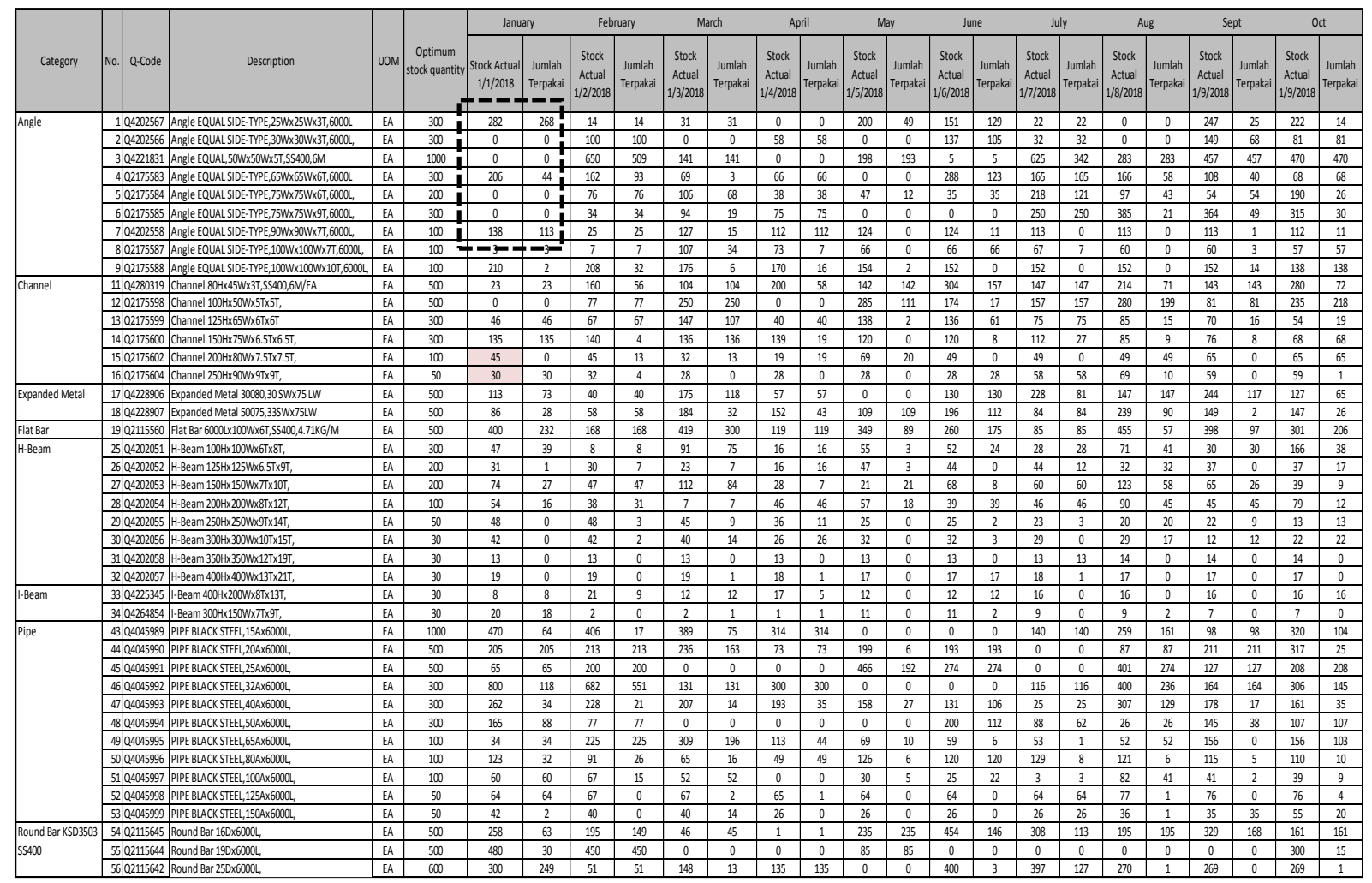

Source: KP company data 2018

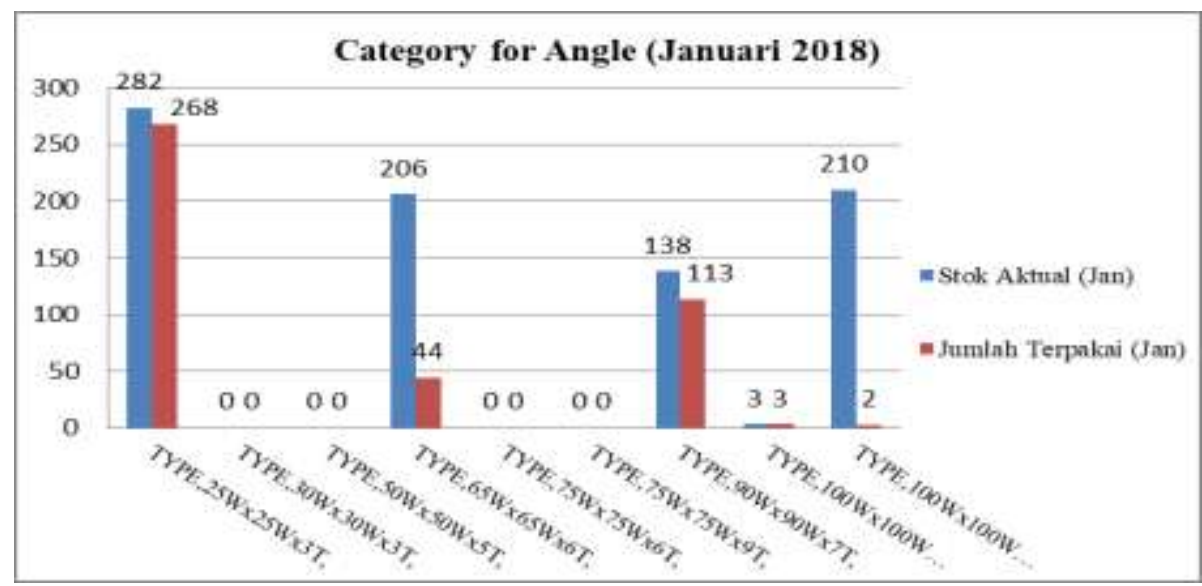

Chart 1. Example of the Angle category stock for January 2018 
Table 1. shows an imbalance between the actual stock and the amount of usage as well as the optimum estimate of stock quantity which is only based on previous usage experience. In addition, in certain months and certain types of materials are often out of stock so that the process of repairing facilities or components of the factory is often constrained so that it can hamper the smooth production process. Therefore this study wants to assist companies in planning optimal inventory control of steel material in the hope of ensuring the needs and smoothness of company activities in the right quantity and quality as well as at optimal costs.

PT. Krakatau Posco has not used the correct method of controlling steel raw material fabrication, therefore this research will use the economic order quantity (EOQ) method of inventory control one of the most appropriate and can be more effective and efficient so that the steel material needs can be met all. The purpose of controlling raw materials is to find out (1) optimal quantity every time in purchasing raw materials, (2) points that indicate the time to reorder (ROP), (3) maximum inventory, and (4) total cost raw material inventory (total inventory cost) to avoid the risk of running out and also excess raw materials so as to minimize the cost of steel material fabrication.

\section{LITERATURE REVIEW Inventory}

Inventories are defined as goods, materials, or assets owned by the company for future use. Inventories are available for sale in normal business activities, in the production process and or in travel and in the form of materials or supplies to be used in the production process or in providing services (Indonesian Institute of Accountants 2014: SFAS No. 14)

\section{Inventory Control}

Inventory control is a model commonly used to solve problems associated with controlling raw materials and finished goods in a company's activities. The distinctive feature of the inventory model is that the optimal solution is focused on guaranteeing supplies at the lowest possible cost.

Meanwhile, to make savings in the supply of supplies and also the smooth production process must be controlled inventory as mentioned by Agus Ristono (2013: 4) "An inventory control carried out by a company certainly has certain objectives. Inventory control carried out is to maintain inventory levels at optimal levels so that savings are obtained for these inventories.

\section{Purpose of Inventory Control}

The purpose of inventory control is to obtain the right quality and quantity of materials or items available at the time needed with minimum costs for the benefit or benefit of the company. Ristono (2013), detailed inventory control objectives are an effort to:

1. Keeping the company from running out of inventory so that it can result in the cessation of production activities

2. Keeping the formation of inventory by the company is not too large or excess

3. Keeping small purchases can be avoided because this will resulting in order costs too large.

\section{ABC Analysis}

$A B C$ analysis divides inventory into three classes based on the value (value) generated by the inventory (Schroeder, 2010). This principle teaches to focus inventory 
control on the types of inventory that are of high or critical value rather than those of low value or trivial. According to Schroeder (2010), ABC classification is as follows:

1. Class A is goods that provide high value. Even though group A is only represented by $20 \%$ of the total inventory available, the value given is $80 \%$.

2. Class B is goods that give a medium value. This class B inventory group is represented by $30 \%$ of the total inventory and the resulting value is $15 \%$.

3. Class $\mathrm{C}$ is goods which give low value. The $\mathrm{C}$ class inventory group is represented by $50 \%$ of the total available inventory and the value generated is $5 \%$.

\section{Economic Order Quantity (EOQ) Method}

Economic Order Quantity (EOQ) is the quantity of material purchased at each purchase with the most minimal cost. The EOQ method can be used for both purchased and self-produced items (Handoko 2011: 340). Meanwhile, according to Heizer and Render (2010: 92), EOQ is one of the oldest and most widely known inventory control techniques, this inventory management method answers 2 (two) important questions, when to order and how much to order.

In applying the EOQ method there are several costs that must be considered in determining the purchase amount or profit, including:

1. Order Cost

Order costs represent costs that will be directly related to the order activities of the company. Order costs also consist of opportunity costs (Opportunity Cost). For example, time wasted processing orders, running administrative orders and so on.

According to (Heizer and Render 2010: 94) the formula for ordering costs is as following:

$$
\text { Order Cost }=\frac{D}{Q} \times S
$$

explanation:

$\mathrm{D}=$ Number of needs, units per year

$\mathrm{Q}=$ The number of messages each time

$\mathrm{S}=$ Order fee every time you order

2. Storage costs

Storage costs are costs that must be borne by the company in connection with the existence of raw materials stored in the company. According to (Heizer and Render 2010: 95) storage costs are formulated as follows:

Explanation :

$$
\text { Storage cost }=\frac{Q}{2} x H
$$

$\mathrm{Q}=$ Number of items each time ordered

$\mathrm{H}=$ Storage costs, units per year

Next determine the total cost of supplies (TIC) by adding up the cost of the message and the cost of saving. The formula is as follows (Heizer and Render 2010: 97):

explanation:

$$
T I C=\frac{D}{Q} S+\frac{Q}{2} H
$$

TIC $=$ Total inventory cost

$\mathrm{D}=$ Number of needs, units per year

$\mathrm{Q}=$ Number of items each time ordered

$\mathrm{S}=$ Cost of the message each time a message 
$\mathrm{H}=$ Storage costs, units per year

$Q^{*}=\sqrt{\frac{2 D S}{H}}$

explanation :

$\mathrm{Q}^{*}=$ Optimal Q value

$\mathrm{S}=$ Order fee (rupiah / order)

$\mathrm{H}=$ Storage fee (rupiah / unit / year)

$\mathrm{Q} *$ indicates that the $\mathrm{Q}$ value is optimal, known as the EOQ method

\section{Safety Stock}

A safety stock is an additional inventory held to protect or protect the possibility of a material shortage (stock out). According to (Sofjan Assauri, 2011) the possibility of a stock out can be caused by the use of raw materials that are greater than originally estimated or the delay in the arrival of raw materials ordered.

To determine the cost of safety stock, statistical analysis is used, which is to consider the deviations that have occurred between the estimated use of raw materials and actual use so that the standard deviation is known.

Adapun rumus standar deviasi adalah sebagai berikut :

$$
\begin{gathered}
S D=\sqrt{\frac{\sum(x-\bar{x})^{2}}{n}} \\
S S=S D x Z
\end{gathered}
$$

\section{Reorder Point}

Reorder Point (ROP) is when another order has to be held so that the receipt of the ordered material right at the time of inventory above the safety stock is zero. (According to Martono and Harjito.2011).

According to Heizer and Render (2010: 99) ROP is the level of inventory where when inventory has reached that level, orders must be made immediately and ROP can be calculated with the following formula:

$$
R O P=(D \times L)+S S
$$

\section{Total Company Inventory Costs}

The calculation of total company costs can be calculated using the following formula:

$$
\text { TIC }_{\text {per }}=(\bar{D} \times H)+(n \times S)
$$

\section{RESEARCH METODE}

In this study after the researchers obtained secondary data, the researchers conducted the $\mathrm{ABC}$ method to obtain a category of steel material fabrication category A. After that the researchers used an analysis tool to be able to find out the number of orders that the company 
should have made so as not to be inadequate and not excessive. The analysis tool is inventory control with the economic order quantity (EOQ) method.

The Economic Order Quantity method is used to determine the quantity of inventory orders that minimize direct costs, inventory storage and inventory ordering costs. After doing an economic calculation with EOQ, then calculate the reorder point (ROP), safety stock (SS) and the calculation of the company's total inventory cost (TIC). The final step is to compare the results of economic order quantities between orders placed by the company with the economic order quantity (EOQ)

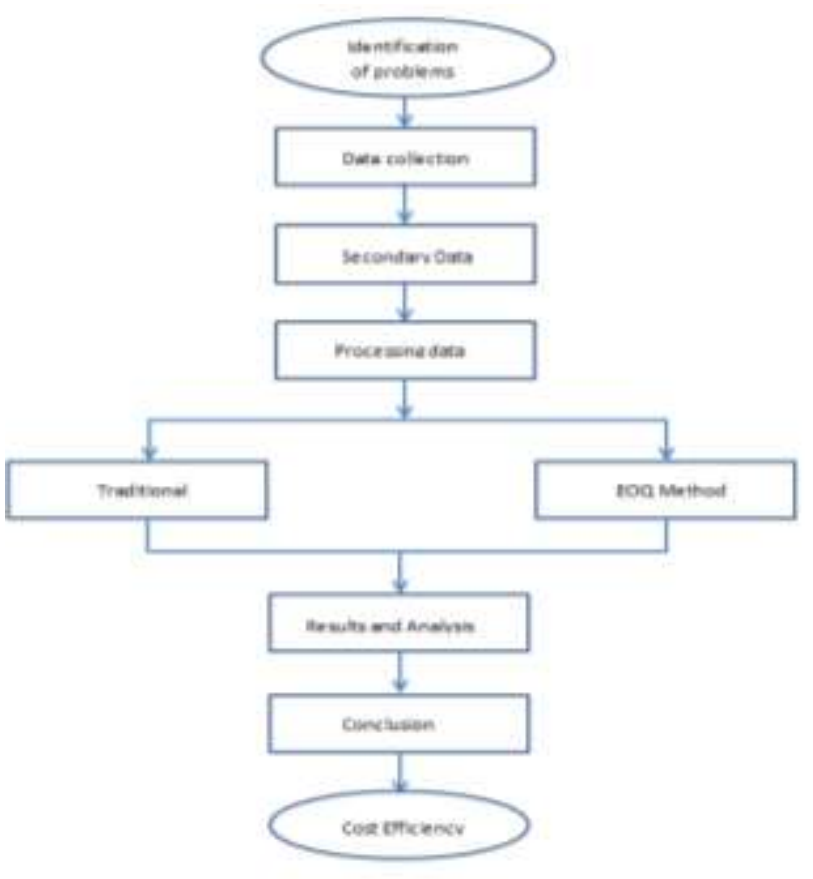

\section{RESEARCH RESULT AND DISCUSSION}

$\mathrm{ABC}$ analysis of Steel Material Fabrication

$\mathrm{ABC}$ analysis for steel material fabrication includes eight raw materials used, consisting of angles, channels, expanded metal, flat bars, H-beams, I-beams, pipes and round bars. By using ABC Analysis, we can find out the classification of raw materials of each type that has critical properties. The critical nature here means which raw materials represent the highest use of money. ABC analysis using manual calculations in Microsoft Excel can be seen in the table below.

\section{Table 2. Analysis ABC of Steel Material Fabrication Type Angle}

\begin{tabular}{|c|c|c|c|c|c|c|c|c|}
\hline Kategori & Tahun & Deskripsi & Total Pemakaian bahan (Unit) & Harga/ Unit (\$) & Total Harga Pemakaian bahan (\$) & Persentase dari \$ - Vol & Komulatif \$ - Vol \$ & Kategori \\
\hline \multirow{28}{*}{ Angle } & \multirow{10}{*}{2016} & \begin{tabular}{|l|l|l}
1 & Angle EQUAL SIDE-TYPE,90Wx90Wx7T,6000L,KSD3503,S5400,9.59KG/M, \\
\end{tabular} & 1163 & 38,55 & 44830,11 & 31,25 & 31,25 & A \\
\hline & & \begin{tabular}{|l|l}
2 Angle EQUAL SIDE-TYPE,75Wx75Wx9T,6000L,KSD3503,S5400,9.96KG/M \\
\end{tabular} & 750 & 34,02 & 25515,26 & 17,79 & 49,04 & A \\
\hline & & 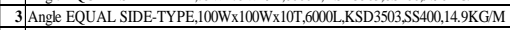 & 351 & 60,36 & 21185,83 & 14,77 & 63,81 & $\mathrm{~A}$ \\
\hline & & \begin{tabular}{|l|l|l}
4 & Angle EQUAL SIDE-TYPE,75Wx75Wx6T,6000L,KSD3503,S5400,6.85KG/M \\
\end{tabular} & 654 & 24,66 & 16125,89 & 11,24 & 75,05 & A \\
\hline & & \begin{tabular}{|l|l|l|l}
5 & Angle EQUAL $50 \mathrm{~W} x 50 \mathrm{~W} \times 5 \mathrm{~T}, \mathrm{SS} 400,6 \mathrm{M}$ \\
\end{tabular} & 1942 & 7,11 & 13803,19 & 9,62 & 84,68 & B \\
\hline & & \begin{tabular}{|c|c|c|c|c|}
$\mathbf{6}$ & AnguL SIDE-TYPE,65Wx65Wx6T,6000L,KSD3503,SS400,5.91KG/M \\
\end{tabular} & 802 & 12,76 & 10230,76 & 7,13 & 91,81 & B \\
\hline & & 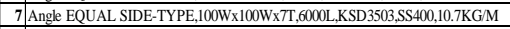 & 183 & 44,02 & 8055,60 & 5,62 & 97,42 & $\mathrm{C}$ \\
\hline & & \begin{tabular}{|l|l|l|l}
8 Angle EQUAL SIDE-TYPE,25Wx25Wx3T,6000L,KSD3503,SS400,1.12KG/M, \\
\end{tabular} & 700 & 3,45 & 2415,13 & 1,68 & 99,11 & C \\
\hline & & \begin{tabular}{|l|l|l|l} 
Angle EQUAL SIDE-TYPE,30Wx30Wx3T,6000L,KSD3503,S5400,1.36KG/M, \\
\end{tabular} & 379 & 3,38 & 1280,19 & 0,89 & 100,00 & $\mathrm{C}$ \\
\hline & & Total & & & 143441,97 & & & \\
\hline & \multirow{10}{*}{2017} & \begin{tabular}{|l|l}
1 Angle EQUAL SIDE-TYPE,90Wx90Wx7T,6000L,KSD3503,S5400,9.59KG/M, \\
\end{tabular} & 1329 & 38,55 & 51228,90 & 25,28 & 25,28 & A \\
\hline & & \begin{tabular}{|l|l|l|} 
Angle EQUAL SIDE-TYPE,75Wx75Wx9T,6000L,KSD3503,SS400,9.96KG/M \\
\end{tabular} & 1002 & 34,02 & 34088,39 & 16,82 & 42,10 & A \\
\hline & & \begin{tabular}{|l|l|l|l|l} 
Angle EQUAL SIDE-TYPE,100Wx100Wx10T,6000L,KSD3503,SS400,14.9KG/M \\
\end{tabular} & 460 & 60,36 & 27764,91 & 13,70 & 55,80 & $\mathrm{~A}$ \\
\hline & & \begin{tabular}{|l|l|l|l}
4 & Angle EQUAL SIDE-TYPE,65Wx65Wx6T,6000L,KSD3503,SS400,5.91KG/M \\
\end{tabular} & 1858 & 12,76 & 23701,69 & 11,70 & 67,49 & A \\
\hline & & \begin{tabular}{|l|l|} 
5 Angle EQUAL SIDE-TYPE,75Wx75Wx6T,6000L,KSD3503,SS400,6.85KG/M \\
\end{tabular} & 898 & 24,66 & 22142,28 & 10,93 & 78,42 & $\mathrm{~A}$ \\
\hline & & \begin{tabular}{|l|l|l|l|l}
6 & Angle EQUAL,50Wx50WxsT,SS400,6M \\
\end{tabular} & 3039 & 7,11 & 21600,35 & 10,66 & 89,08 & B \\
\hline & & \begin{tabular}{|l|l|}
7 Angle EQUAL SIDE-TYPE,100Wx100W xTT,6000L,KSD3503,SS400,10.7KG/M \\
\end{tabular} & 390 & 44,02 & 17167,67 & 8,47 & 97,55 & $\mathrm{C}$ \\
\hline & & \begin{tabular}{|l|l|l|l}
8 Angle EQUAL SIDE-TYPE,25Wx25Wx3T,6000L,KSD3503,SS400,1.12KG/M, \\
\end{tabular} & 931 & 3,45 & 3212,13 & 1,58 & 99,13 & C \\
\hline & & \begin{tabular}{|c|c|c|c|}
9 & Angle EQUAL SIDE-TYPE,30Wx30Wx3T,6000L,KSD3503,SS400,1.36KG/M, \\
\end{tabular} & 520 & 3,38 & 1756,47 & 0,87 & 100,00 & $\mathrm{C}$ \\
\hline & & Total & & & 202662,79 & & & \\
\hline & \multirow{8}{*}{2018} & \begin{tabular}{|l|l|l|l|l|} 
Angle EQUAL SIDE-TYPE,100Wx100Wx10T,6000L,KSD3503,SS400,14.9KG/M \\
\end{tabular} & 355 & 60,36 & 21427,27 & 20,29 & 20,29 & A \\
\hline & & \begin{tabular}{|l|l|l|l}
2 & Angle EQUAL, $50 \mathrm{~W} x 50 \mathrm{~W} \times 5 \mathrm{~T}, \mathrm{SS} 400,6 \mathrm{M}$ \\
\end{tabular} & 2523 & 7,11 & 17932,77 & 16,98 & 37,27 & A \\
\hline & & \begin{tabular}{|c|c|c|c|c|}
3 & AngUAL SIDE-TYPE,75Wx75Wx9T,6000L,KSD3503,SS400,9.96KG/M \\
\end{tabular} & 482 & 34,02 & 16397,81 & 15,53 & 52,79 & $\mathrm{~A}$ \\
\hline & & \begin{tabular}{|l|l|l|} 
4 Angle EQUAL SIDE-TYPE,75Wx75Wx6T,6000L,KSD3503,S5400,6.85KG/M \\
\end{tabular} & 637 & 24,66 & 15706,72 & 14,87 & 67,66 & A \\
\hline & & \begin{tabular}{|c|c|c|c|} 
Angle EQUAL SIDE-TYPE,90Wx90Wx7T,6000L,KSD3503,S4400,9.59KG/M, \\
\end{tabular} & 288 & 38,55 & 11101,52 & 10,51 & 78,18 & A \\
\hline & & \begin{tabular}{|l|l|l|}
6 & Angle EQUAL SIDE-TYPE,65Wx65Wx6T,6000L,KSD3503,S5400,5.91KG/M \\
\end{tabular} & 760 & 12,76 & 9694,99 & 9,18 & 87,36 & $\mathrm{~B}$ \\
\hline & & \begin{tabular}{|l|l|}
7 & Angle EQUAL SIDE-TYPE,100Wx100W xTT,6000L,KSD3503,SS400,10.7KG/M \\
\end{tabular} & 196 & 44,02 & 8627,86 & 8,17 & 95,52 & $\mathrm{C}$ \\
\hline & & \begin{tabular}{|l|l|l|l|l}
8 Angle EQUAL SIDE-TYPE,25Wx25Wx3T,6000L,KSD3503,SS400,1.12KG/M, \\
\end{tabular} & 767 & 3,45 & 2646,30 & 2,51 & 98,03 & C \\
\hline
\end{tabular}


Table 3. Analysis ABC of Steel Material Fabrication Type Pipe

\begin{tabular}{|c|c|c|c|c|c|c|c|c|}
\hline Kategori & Tahun & Deskripsi & Total Pemakaian bahan (Unit) & Harga/ Unit (\$) & \begin{tabular}{|l} 
Total Harga Pemakaian bahan $(\$)$ \\
\end{tabular} & Persentase dari \$ - Vol & Komulatif \$ - Vol \$ & Kategori \\
\hline \multirow{35}{*}{ Pipe } & \multirow{12}{*}{2016} & \begin{tabular}{l|l} 
PIPE BLACK STEEL,32Ax6000L,KSD3507,SPP,3.16KG/M \\
\end{tabular} & 1618 & 10,69 & 17296,18 & 15,58 & 15,58 & $\mathrm{~A}$ \\
\hline & & 2 PIPE BLACK STEEL,50Ax6000L,KSD3507,SPP,5.12KG/M & 1075 & 15,30 & 16444,01 & 14,81 & 30,40 & $\mathrm{~A}$ \\
\hline & & \begin{tabular}{l|l|}
3 PIPE BLACK STEEL,80Ax6000L,KSD3507,SPP,8.49KG/M \\
\end{tabular} & 489 & 29,03 & 14195,59 & 12,79 & 43,19 & $\mathrm{~A}$ \\
\hline & & 4 PIPE BLACK STEEL,25Ax6000L,KSD3507,SPP,2.45KG/M & 1554 & 8,34 & 12957,38 & 11,67 & 54,86 & A \\
\hline & & 5 PIPE BLACK STEEL,150Ax6000L,KSD3507,SPP,19.2KG/M & 184 & 67,00 & 12328,00 & 11,11 & 65,96 & $\mathrm{~A}$ \\
\hline & & \begin{tabular}{l|l|} 
PIPE BLACK STEEL,40Ax6000L,KSD3507,SPP,3.63KG/M \\
\end{tabular} & 890 & 12,07 & 10743,52 & 9,68 & 75,64 & A \\
\hline & & \begin{tabular}{|l|l|l|} 
PIPE BLACK STEEL,20Ax6000L,KSD3507,SPP,1.60KG/M \\
\end{tabular} & 1780 & 4,83 & 8600,04 & 7,75 & 83,39 & $\mathrm{~B}$ \\
\hline & & \begin{tabular}{|l|l|}
8 PIPE BLACK STEEL,65Ax6000L,KSD3507,SPP,6.34KG/M \\
\end{tabular} & 301 & 20,78 & 6254,72 & 5,63 & 89,03 & B \\
\hline & & \begin{tabular}{|l|l|l|l|}
9 IPE BLACK STEEL $15000 L, K S D 3507, S P P, 1.25 K G / M$ \\
\end{tabular} & 1349 & 3,72 & 5021,98 & 4,52 & 93,55 & $\mathrm{~B}$ \\
\hline & & \begin{tabular}{|l|l|l|l|}
10 PIPE BLACK STEEL,100Ax6000L,KSD3507,SPP,12.2KG/M \\
\end{tabular} & 103 & 42,08 & 4334,56 & 3,90 & 97,46 & $\mathrm{C}$ \\
\hline & & \begin{tabular}{|l|}
$11 \mid$ PIPE BLACK STEEL,125Ax6000L,KSD3507,SPP,16.1KG/M \\
\end{tabular} & 50 & 56,50 & 2824,80 & 2,54 & 100,00 & $\mathrm{C}$ \\
\hline & & Total & & & 111000,79 & & & \\
\hline & \multirow{12}{*}{2017} & \begin{tabular}{l|l|l|l|} 
PIPE BLACK STEEL,125Ax6000L,KSD3507,SPP,16.1KG/M \\
\end{tabular} & 317 & 56,50 & 17909,24 & 14,72 & 14,72 & $\mathrm{~A}$ \\
\hline & & \begin{tabular}{|l|l|l|}
2 PIPE BLACK STEEL,25Ax6000L,KSD3507,SPP,2.45KG/M \\
\end{tabular} & 1924 & 8,34 & 16042,47 & 13,18 & 27,90 & A \\
\hline & & \begin{tabular}{|l|l|l|} 
PIPE BLACK STEEL,150Ax6000L,KSD3507,SPP,19.2KG/M \\
\end{tabular} & 212 & 67,00 & 14204,00 & 11,67 & 39,57 & A \\
\hline & & \begin{tabular}{|l|l|l|}
4 IPE BLACK STEEL,65Ax6000L,KSD3507,SPP,6.34KG/M \\
\end{tabular} & 673 & 20,78 & 13984,80 & 11,49 & 51,06 & $\mathrm{~A}$ \\
\hline & & \begin{tabular}{|l|l|l|} 
PIPE BLACK STEEL,100Ax6000L,KSD3507,SPP,12.2KG/M \\
\end{tabular} & 270 & 42,08 & 11362,43 & 9,34 & 60,40 & A \\
\hline & & $\begin{array}{l}\text { 6IPE BLACK STEEL,80Ax6000L,KSD3507,SPP,8.49KG/M } \\
\end{array}$ & 340 & 29,03 & 9870,15 & 8,11 & 68,51 & A \\
\hline & & \begin{tabular}{|l|l|}
7 PIPE BLACK STEEL,32Ax6000L,KSD3507,SPP,3.16KG/M \\
\end{tabular} & 917 & 10,69 & 9802,59 & 8,06 & 76,57 & A \\
\hline & & \begin{tabular}{|l|l|}
8 PIPE BLACK STEEL,50Ax6000L,KSD3507,SPP,5.12KG/M \\
\end{tabular} & 621 & 15,30 & 9499,29 & 7,81 & 84,37 & B \\
\hline & & \begin{tabular}{l|l|}
9 & PIPE BLACK STEEL,40Ax6000L,KSD3507,SPP,3.63KG/M \\
\end{tabular} & 724 & 12,07 & 8739,67 & 7,18 & 91,55 & B \\
\hline & & \begin{tabular}{|l|l|} 
PIPE BLACK STEEL,20Ax6000L,KSD3507,SPP,1.60KG/M \\
\end{tabular} & 1218 & 4,83 & 5884,75 & 4,84 & 96,39 & $\mathrm{C}$ \\
\hline & & 11|PIPE BLACK STEEL,15Ax6000L,KSD3507,SPP,1.25KG/M & 1180 & 3,72 & 4392,83 & 3,61 & 100,00 & $\mathrm{C}$ \\
\hline & & Total & & & 121692,23 & & & \\
\hline & \multirow{11}{*}{2018} & \begin{tabular}{l|l|l|l|} 
PIPE BLACK STEEL,32Ax6000L,KSD3507,SPP,3.16KG/M \\
\end{tabular} & 1922 & 10,69 & 20545,90 & 18,78 & 18,78 & A \\
\hline & & \begin{tabular}{|l|l|l|}
2 PIPE BLACK STEEL,65Ax6000L,KSD3507,SPP,6.34KG/M \\
\end{tabular} & 724 & 20,78 & 15044,57 & 13,75 & 32,54 & A \\
\hline & & 3 PIPE BLACK STEEL,25Ax6000L,KSD3507,SPP,2.45KG/M & 1738 & 8,34 & 14491,59 & 13,25 & 45,79 & A \\
\hline & & 4 PIPE BLACK STEEL,100Ax6000L,KSD3507,SPP,12.2KG/M & 228 & 42,08 & 9594,94 & 8,77 & 54,56 & A \\
\hline & & \begin{tabular}{|l|l|l|} 
PIPE BLACK STEEL,50Ax6000L,KSD3507,SPP,5.12KG/M \\
\end{tabular} & 566 & 15,30 & 8657,96 & 7,92 & 62,47 & A \\
\hline & & \begin{tabular}{|c|c|c|} 
PIPE BLACK STEEL,80Ax6000L,KSD3507,SPP,8.49KG/M \\
\end{tabular} & 292 & 29,03 & 8476,71 & 7,75 & 70,22 & A \\
\hline & & 7 PIPE BLACK STEEL,125Ax6000L,KSD3507,SPP,16.1KG/M & 136 & 56,50 & 7683,46 & 7,02 & 77,25 & A \\
\hline & & \begin{tabular}{|l|l|} 
PIPE BLACK STEEL,150Ax6000L,KSD3507,SPP,19.2KG/M \\
\end{tabular} & 105 & 67,00 & 7035,00 & 6,43 & 83,68 & $\mathrm{~B}$ \\
\hline & & \begin{tabular}{l|l|} 
PIPE BLACK STEEL,40Ax6000L,KSD3507,SPP,3.63KG/M \\
\end{tabular} & 569 & 12,07 & 6868,61 & 6,28 & 89,96 & $\mathrm{~B}$ \\
\hline & & \begin{tabular}{|l|l|}
10 PIPE BLACK STEEL,20Ax6000L,KSD3507,SPP,1.60KG/M \\
\end{tabular} & 1357 & 4,83 & 6556,33 & 5,99 & 95,95 & $\mathrm{C}$ \\
\hline & & 11 PIPE BLACK STEEL,15Ax6000L,KSD3507,SPP,1.25KG/M & 1189 & 3,72 & 4426,34 & 4,05 & 100,00 & $\mathrm{C}$ \\
\hline
\end{tabular}

Table 4. Analysis ABC of Steel Material Fabrication Type H-Beam

\begin{tabular}{|c|c|c|c|c|c|c|c|c|}
\hline Kategori & Tahun & Deskripsi & Total Pemakaian bahan (Unit) & Harga/ Unit (\$) & Total Harga Pemakaian bahan (\$) & Persentase dari \$ - Vol & Komulatif \$ - Vol \$ & Kategori \\
\hline \multirow{27}{*}{ H - Beam } & \multirow{9}{*}{2016} & \begin{tabular}{l|l}
1 & H-Beam 150Hx150Wx7Tx10T,KSD3503,SS400,31.5KG/M,12M
\end{tabular} & 483 & 213,05 & 102905,52 & 20,42 & 20,42 & $\mathrm{~A}$ \\
\hline & & $2 / \mathrm{H}-$ Beam $125 \mathrm{Hx} 125 \mathrm{~W} \times 65 \mathrm{~T} \times 9 \mathrm{~T}$ KSD $35025 \mathrm{SS} 400238 \mathrm{KG} / \mathrm{M} / 2 \mathrm{M}$ & 520 & 197.11 & 10249483 & 2034 & 4077 & A \\
\hline & & 3 H-Beam $200 \mathrm{H} \times 200 \mathrm{~W} \times 8 \mathrm{~T} \times 12 \mathrm{~T}, \mathrm{KSD} 3503, \mathrm{SS} 400,49.9 \mathrm{KG} / \mathrm{M}, 12 \mathrm{M}$ & 245 & 410,44 & 100559,00 & 19,96 & 60,72 & $\mathrm{~A}$ \\
\hline & & \begin{tabular}{c|c|}
4 & H-Beam 100Hx100Wx6Tx8T,KSD3502,SS400,17.2KG/M,12M \\
\end{tabular} & 500 & 133,98 & 66988,63 & 13,30 & 74,02 & A \\
\hline & & \begin{tabular}{c|c|c|}
5 & $\mathrm{H}-$B eam $400 \mathrm{H} \times 400 \mathrm{~W} \times 13 \mathrm{~T} \times 21 \mathrm{~T}, \mathrm{KSD} 3503, \mathrm{SS} 400,233 \mathrm{KG} / \mathrm{M}, 12 \mathrm{M}$ \\
\end{tabular} & 42 & 1100,00 & 46200,00 & 9,17 & 83,19 & B \\
\hline & & 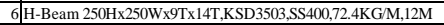 & 77 & 493,01 & 37961,40 & 7,53 & 90,72 & B \\
\hline & & 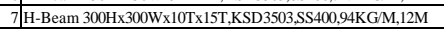 & 40 & 618,40 & 24735,88 & 4,91 & 95,63 & $\mathrm{C}$ \\
\hline & & $8 \mid \mathrm{H}$-Beam 350H×350Wx12Tx19T,KSD3503,SS400,137KG/M,12M & 16 & 1376,00 & 22016,02 & 4,37 & 100,00 & $\mathrm{C}$ \\
\hline & & Total & & & 503861,28 & & & \\
\hline & \multirow{9}{*}{2017} & \begin{tabular}{l|l}
1 & H-Beam 200Hx200Wx8Tx12T,KSD3503,SS400,49.9KG/M,12M \\
\end{tabular} & 361 & 410,44 & 148170,61 & 26,19 & 26,19 & A \\
\hline & & \begin{tabular}{l|l|l} 
2 H-Beam 125Hx125Wx6.5T×9T,KSD3502,SS400,23.8KG/M,12M \\
\end{tabular} & 371 & 197,11 & 73126,12 & 12,93 & 39,12 & A \\
\hline & & \begin{tabular}{l|l|l}
3 & H-Beam 400H×400Wx13T×21T,KSD3503,SS400,233KG/M,12M \\
\end{tabular} & 59 & 1100,00 & 64900,00 & 11,47 & 50,59 & A \\
\hline & & 4/H-Beam 250Hx250Wx9Tx14T,KSD3503,SS400,72.4KG/M,12M & 127 & 493,01 & 62611,66 & 11,07 & 61,66 & $\mathrm{~A}$ \\
\hline & & 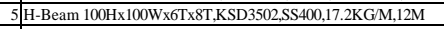 & 446 & 133,98 & 59753,86 & 10,56 & 72,22 & A \\
\hline & & 6 H-Beam 300H×300Wx10Tx15T,KSD3503,SS400,94KG/M,12M & 92 & 618,40 & 56892,51 & 10,06 & 82,28 & B \\
\hline & & \begin{tabular}{c|c|}
7 H-Beam 350Hx350Wx12Tx19T,KSD3503,SS400,137KG/M,12M \\
\end{tabular} & 38 & 1376,00 & 52288,05 & 9,24 & 91,53 & B \\
\hline & & \begin{tabular}{|l|l|}
8 H-Beam 150Hx150Wx7Tx10T,KSD3503,SS400,31.5KG/M,12M \\
\end{tabular} & 225 & 213,05 & 47937,35 & 8,47 & 100,00 & C \\
\hline & & Total & & & 565680,16 & & & \\
\hline & \multirow{9}{*}{2018} & \begin{tabular}{l|l|l|l|}
1 & H-Beam 200Hx200Wx8Tx12T,KSD3503,SS400,49.9KG/M,12M
\end{tabular} & 372 & 410,44 & 152685,50 & 28,22 & 28,22 & A \\
\hline & & 2 H-Beam 150Hx150Wx7Tx10T,KSD3503,SS400,31.5KG/M,12M & 377 & 213,05 & 80321,70 & 14,84 & 43,06 & A \\
\hline & & \begin{tabular}{c|c|c|}
3 H-Beam 300Hx300Wx10Tx15T,KSD3503,SS400,94KG/M,12M \\
\end{tabular} & 126 & 618,40 & 77918,01 & 14,40 & 57,46 & A \\
\hline & & \begin{tabular}{c|c|c|c|}
4 & H-Beam 400Hx400Wx13T×21T,KSD3503,SS400,233KG/M,12M \\
\end{tabular} & 55 & 1100,00 & 60500,00 & 11,18 & 68,64 & A \\
\hline & & 5 H-Beam 100Hx100W $\mathrm{W} 6 \mathrm{~T} \times 8 \mathrm{~T}, \mathrm{KSD} 3502, \mathrm{SS} 400,17.2 \mathrm{KG} / \mathrm{M}, 12 \mathrm{M}$ & 430 & 133,98 & 57610,22 & 10,65 & 79,29 & A \\
\hline & & \begin{tabular}{c|c|c|}
6 & H-Beam 250Hx250Wx9Tx14T,KSD3503,SS400,72.4KG/M,12M
\end{tabular} & 106 & 493,01 & 52258,55 & 9,66 & 88,95 & B \\
\hline & & 7 H-Beam 350H×350Wx12Tx19T,KSD3503,SS400,137KG/M,12M & 27 & 1376,00 & 37152,04 & 6,87 & 95,81 & $\mathrm{C}$ \\
\hline & & $8 \mid \mathrm{H}$-Beem 125Hx125W $\times 6.5 T \times 9 T, K S D 3502, S S 400,23.8 \mathrm{KG} / \mathrm{M}, 12 \mathrm{M}$ & 115 & 197,11 & 22667,13 & 4,19 & 100,00 & C \\
\hline & & Total & & & 541113,14 & & & \\
\hline
\end{tabular}

Table 5. Analysis ABC of Steel Material Fabrication Type channel

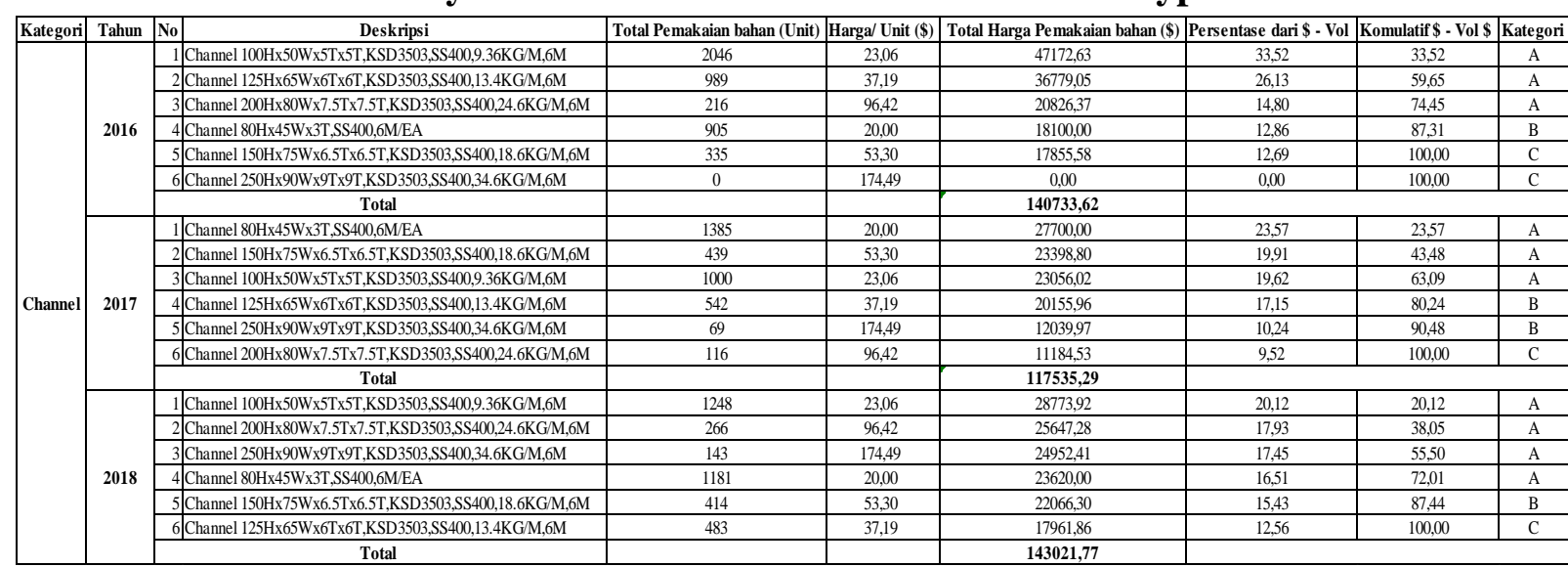


Table 6. Analysis ABC of Steel Material Fabrication Type round bar

\begin{tabular}{|c|c|c|c|c|c|c|c|c|}
\hline Kategori & Tahun 1 & Deskripsi & Total Pemakaian bahan (Unit) & Harga/ Unit (\$) & Total Harga Pemakaian bahan (\$) & Persentase dari \$ - Vol & Komulatif \$ - Vol \$ & Kategori \\
\hline \multirow{13}{*}{ Round Bar } & \multirow{4}{*}{2016} & \begin{tabular}{l|l}
$1 \mid$ & Round Bar 25Dx6000L,KSD3503,SS400,3.85KG/M \\
\end{tabular} & 1132 & 96,42 & 109145,58 & 65,71 & 65,71 & A \\
\hline & & \begin{tabular}{|l|l|} 
Round Bar 19Dx6000L,KSD3503,SS400,2.23KG/M \\
\end{tabular} & 1378 & 37,19 & 51245,22 & 30,85 & 96,56 & $\mathrm{~B}$ \\
\hline & & \begin{tabular}{l|l|}
3 & Round Bar 16Dx6000L,KSD3503,S5400,1.58KG/M \\
\end{tabular} & 248 & 23,06 & 5717,89 & 3,44 & 100,00 & $\mathrm{C}$ \\
\hline & & Total & & & 166108,70 & & & \\
\hline & \multirow{5}{*}{2017} & & & & & & & \\
\hline & & 1 Round Bar 19Dx6000L,KSD3503,SS400,2.23KG/M & 746 & 53,30 & 39761,97 & 49,23 & 49,23 & A \\
\hline & & \begin{tabular}{l|l|}
2 & Round Bar 25Dx6000L,KSD3503,SS400,3.85KG/M \\
\end{tabular} & 1274 & 23,06 & 29373,38 & 36,36 & 85,59 & B \\
\hline & & \begin{tabular}{|l|} 
Round Bar 16Dx6000L,KSD3503,SS400,1.58KG/M \\
\end{tabular} & 582 & 20,00 & 11640,00 & 14,41 & 100,00 & $\mathrm{C}$ \\
\hline & & Total & & & 80775,35 & & & \\
\hline & \multirow{4}{*}{2018} & \begin{tabular}{l|l}
1 & Round Bar 25Dx6000L,KSD3503,SS400,3.85KG/M
\end{tabular} & 650 & 21,64 & 14066,65 & 38,61 & 38,61 & A \\
\hline & & 2 Round Bar 16Dx6000L,KSD3503,SS400,1.58KG/M & 1647 & 7,16 & 11792,52 & 32,37 & 70,97 & A \\
\hline & & \begin{tabular}{l|l}
3 & Round Bar 19Dx6000L,KSD3503,SS400,2.23KG/M \\
\end{tabular} & 761 & 13,90 & 10576,70 & 29,03 & 100,00 & B \\
\hline & & Total & & & 36435,87 & & & \\
\hline
\end{tabular}

Table 7. Analysis ABC of Steel Material Fabrication Type expanded metal

\begin{tabular}{|c|c|c|c|c|c|c|c|c|}
\hline Kategori & Tahun $\mathrm{N}$ & Deskripsi & Total Pemakaian bahan (Unit) & Harga/Unit (\$) & Total Harga Pemakaian bahan (\$) & Persentase dari \$ - Vol & Komulatif \$-Vol\$ & Kategori \\
\hline \multirow{9}{*}{ Expanded Metal } & \multirow{3}{*}{2016} & 1 Expanded Metal 50075,33SWx75LW,5x7.5,1.2X2.4,LCA,SS400,50, & 1393 & 49,56 & 69041,41 & 75,10 & 75,10 & A \\
\hline & & 2 Expanded Metal 30080,30 SWx75 LW,3x8,1.2X2.4,LCA,SS400,28, & 730 & 31,35 & 22887,88 & 24,90 & 100,00 & $B$ \\
\hline & & Total & & & 91929,29 & & & \\
\hline & \multirow{3}{*}{2017} & 1 Expanded Metal 50075,33SWX75LW,5x7.5,1.2X2.4,LCA,SS400,50, & 1497 & 49,56 & 74195,98 & 61,43 & 61,43 & A \\
\hline & & 2 Expanded Metal 30080,30 SWx75 LW,3x8,1.2X2.4,LCA,SS400,28, & 1486 & 31,35 & 46590,94 & 38,57 & 100,00 & $B$ \\
\hline & & Total & & & 120786,92 & & & \\
\hline & \multirow{3}{*}{2018} & 1 Expanded Metal 50075,33SWX75LW,5x7.5,1.2X2.4,LCA,SS400,50, & 705 & 49,56 & 34941,99 & 53,26 & 53,26 & A \\
\hline & & 2 Expanded Metal 30080,30 SWx75 LW,3x8,1.2X2.4,LCA,SS400,28, & 978 & 31,35 & 30663,48 & 46,74 & 100,00 & $B$ \\
\hline & & Total & & & 65605,48 & & & \\
\hline
\end{tabular}

Table 8. Analysis ABC of Steel Material Fabrication Type I-beam

\begin{tabular}{|c|c|c|c|c|c|c|c|c|}
\hline Kategori & Tahun & Deskripsi & Total Pemakaian bahan (Unit) & Harga/ Unit \$ & Total Harga Pemakaian bahan $(\$)$ & Persentase dari \$ - Vol & Komulatif \$ - Vol \$ & Kategori \\
\hline \multirow{9}{*}{ I Beam } & \multirow{3}{*}{2016} & 1 I-Beam 400H×200Wx8Tx13T,SS400,12M/EA, WIDE FLANGE & 22 & 589,10 & 12960,22 & 87,55 & 87,55 & A \\
\hline & & \begin{tabular}{l|l|} 
I-Beam 300Hx150Wx7Tx9T,SS400,12M, WIDE FLANGE \\
\end{tabular} & 10 & 184,26 & 1842,62 & 12,45 & 100,00 & B \\
\hline & & Total & & & 14802,84 & & & \\
\hline & \multirow{3}{*}{2017} & \begin{tabular}{l|l|l|}
1 -Beam 300Hx150Wx7Tx9T,SS400,12M, WIDE FLANGE \\
\end{tabular} & 232 & 283,52 & 65776,95 & 73,00 & 73,00 & $\mathrm{~B}$ \\
\hline & & \begin{tabular}{|l|l|l|} 
I-Beam 400H $200 \mathrm{~W} \times 8 \mathrm{~T} \times 13 \mathrm{~T}, \mathrm{SS} 400,12 \mathrm{M} / \mathrm{EA}, \mathrm{WIDE}$ FLANGE \\
\end{tabular} & 50 & 486,58 & 24328,80 & 27,00 & 27,00 & A \\
\hline & & Total & & & 90105,74 & & & \\
\hline & \multirow{3}{*}{2018} & 1 I-Beam 400H x200Wx8Tx13T,SS400,12M/EA, WIDE FLANGE & 63 & 486,58 & 30654,28 & 76,43 & 76,43 & A \\
\hline & & \begin{tabular}{l|l}
2 I-Beam 300Hx150Wx7Tx9T,SS400,12M, WIDE FLANGE \\
\end{tabular} & 24 & 393,96 & 9455,02 & 23,57 & 100,00 & B \\
\hline & & Total & & & 40109,31 & & & \\
\hline
\end{tabular}

Table 9. Analysis ABC of Steel Material Fabrication Type flat bar

\begin{tabular}{|c|c|c|c|c|c|c|c|c|}
\hline Kategori & Tahun & Deskripsi & Total Pemakaian bahan (Unit) & Harga/ Unit (\$) & Total Harga Pemakaian bahan $(\$)$ & Persentase dari \$ - Vol & Komulatif \$ - Vol \$ & Kategori \\
\hline \multirow{6}{*}{ Flat Bar } & \multirow{2}{*}{2016} & \begin{tabular}{l|l|}
1 & Flat Bar 6000Lx100Wx6T,SS400,4.71KG/M
\end{tabular} & 1102 & 14,62 & 16107,83 & 100,00 & 100,00 & A \\
\hline & & Total & & & 16107,83 & & & \\
\hline & \multirow{2}{*}{2017} & \begin{tabular}{l|l|l|l|} 
Flat Bar $6000 \mathrm{Lx} 100 \mathrm{~W} 6 \mathrm{~T}, \mathrm{SS} 400,4.71 \mathrm{KG} / \mathrm{M}$
\end{tabular} & 937 & 14,62 & 13696,04 & 100,00 & 100,00 & $\mathrm{~A}$ \\
\hline & & Total & & & 13696,04 & & & \\
\hline & \multirow{2}{*}{2018} & \begin{tabular}{l|l|l|l|} 
Flat Bar $6000 \mathrm{Lx} 100 \mathrm{~W} 6 \mathrm{~T}, \mathrm{SS} 400,4.71 \mathrm{KG} / \mathrm{M}$
\end{tabular} & 1735 & 14,62 & 25360,32 & 100,00 & 100,00 & A \\
\hline & & Total & & & 25360,32 & & & \\
\hline
\end{tabular}

\section{Inventory Cost}

According to Puspita and Suryani (2012), inventory is one aspect that needs to be considered by companies, especially for manufacturing companies. Inventory costs are costs arising from the availability of raw materials. Inventory costs consist of, order costs (Ordering Cost or Setup Cost), and storage costs (Holding Cost).

\begin{tabular}{|c|c|} 
Table 10. Storage cost of steel material fabrication \\
\hline The Cost of Ordering Component & Ordering Cost ( Rp. ) \\
\hline Unloading Costs (Using a Forklift) & 500,000 \\
\hline Total cost & 500,000 \\
\hline
\end{tabular}

Order costs incurred by PT Krakatau Posco only consist of the costs of unloading costs while shipping costs are included in the price of raw materials and are free. So the total booking fee is IDR 500,000. As for the storage costs incurred consisting of the salary of one steel material fabrication operator, loading costs (using a forklift). 
Table 11. Total cost of storing steel material fabrication / year

\begin{tabular}{|c|c|c|}
\hline Storage Cost Component & Cost (Rp.) / Year & Total Cost (Rp.) \\
\hline Salary 1 Operator & $6.000 .000 \times 12$ (Month) & $72,000,000$ \\
\hline Load Costs (Using a Forklift) & $500.000 \times 720$ (Hour) & $360,000,000$ \\
\hline \multicolumn{2}{|c|}{ Total Cost (Rp) } & $432,000,000$ \\
\hline
\end{tabular}

Table 12. Storage Cost Per unit

\begin{tabular}{|c|c|c|c|c|c|c|c|c|c|c|c|c|}
\hline Tahun & $\begin{array}{c}\text { Round Bar } \\
\text { (Unit) }\end{array}$ & $\begin{array}{c}\text { Channel } \\
\text { (Unit) }\end{array}$ & $\begin{array}{c}\text { H-Beam } \\
\text { (Unit) }\end{array}$ & $\begin{array}{c}\text { Fat Bar } \\
\text { (Unit) }\end{array}$ & $\begin{array}{c}\text { - Beam } \\
\text { (Unit) }\end{array}$ & $\begin{array}{c}\text { Expanded Metal } \\
\text { (Unit) }\end{array}$ & $\begin{array}{c}\text { Pipe } \\
\text { (Unit) }\end{array}$ & $\begin{array}{c}\text { Angle } \\
\text { (Unit) }\end{array}$ & $\begin{array}{c}\text { Total } \\
\text { (Unit) }\end{array}$ & $\begin{array}{c}\text { Rata } \\
\text { rata }\end{array}$ & $\begin{array}{c}\text { Total Biaya Penyimpanan steel material } \\
\text { fabrication / Tahun (Rp.) }\end{array}$ & Biaya Penyimpanan / Unit (Rp.) \\
\hline $\mathbf{2 0 1 6}$ & 1132 & 3251 & 1923 & 1102 & 32 & 2123 & 9393 & 6924 & 25880 & 3235 & 432.000 .000 & 16992,43 \\
\hline 2017 & 746 & 2824 & 1719 & 937 & 282 & 2983 & 8396 & 10427 & 28314 & 3539,25 & 432.000 .000 & 15257,47 \\
\hline 2018 & 2297 & 2838 & 1608 & 1735 & 87 & 1683 & 8826 & 6624 & 25698 & 3212,25 & 432.000 .000 & 16810,65 \\
\hline
\end{tabular}

The total storage cost for each unit is IDR 16,692 for raw materials in 2016, IDR 15,257 for raw materials in 2017 and IDR 16,810 for raw materials in 2018.

\section{Calculation of EOQ and Order Frequency}

The following will be attached to the calculation for the calculation of each material category, previously the order fee and storage fee will be submitted for each of the material categories.

Table 13. Calculation of EOQ and order frequency in 2016-2018

\begin{tabular}{|c|c|c|c|c|}
\hline Kategori & Tahum & Deskripsi & EOQ & Frekuensi Pemesanan \\
\hline \multirow{13}{*}{ Angle } & \multirow{4}{*}{2016} & 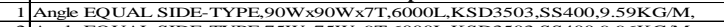 & 263,96 & 4,41 \\
\hline & & 2 Angle EQUAL SIDE-TYPE, $75 \mathrm{~W} \times 75 \mathrm{~W} \times 9 \mathrm{~T}, 6000 \mathrm{~L}, \mathrm{KSD} 3503, \mathrm{SS} 400,9.96 \mathrm{KG} / \mathrm{M}$ & 211,97 & 3,54 \\
\hline & & 3 Angle EQUAL SIDE-TYPE, $100 \mathrm{~W} \times 100 \mathrm{~W} \times 10 \mathrm{~T}, 600 \mathrm{LL}, \mathrm{KSD} 3503, \mathrm{SS} 400,14.9 \mathrm{KG} / \mathrm{M}$ & 145,01 & 2,42 \\
\hline & & 4 Angle EQUAL SIDE-TYPE, $75 \mathrm{~W} \times 75 \mathrm{~W} \times 6 \mathrm{~T}, 6000 \mathrm{~L}, \mathrm{KSD} 3503, \mathrm{SS} 400,6.85 \mathrm{KG} / \mathrm{M}$ & $\frac{197,94}{295,14}$ & $\frac{3,30}{4,50}$ \\
\hline & \multirow{4}{*}{2017} & 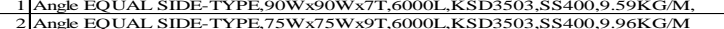 & 295,14 & $\frac{4,50}{3,91}$ \\
\hline & & 3 Angle EQUAL SIDE-TYPE, $100 \mathrm{~W} \times 100 \mathrm{~W} \times 10 \mathrm{~T}, 6000 \mathrm{~L}, \mathrm{KSD} 3503, \mathrm{SS} 400,14.9 \mathrm{KG} / \mathrm{M}$ & $\frac{173,64}{173}$ & $\frac{3,91}{2,65}$ \\
\hline & & 4 Angle EQUAL SIDE-TYPE,65W W65W x6T,6000L,KSD3503,SS400,5.91 KG/M & 348,96 & $\frac{2,05}{5,32}$ \\
\hline & & 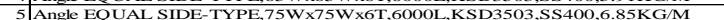 & 242.60 & \\
\hline & \multirow{5}{*}{2018} & 1 Angle EQUAL SIDE-TYPE, $100 \mathrm{~W} \times 100 \mathrm{~W} \times 10 \mathrm{~T}, 600 \mathrm{~L}, \mathrm{KSD} 3503, \mathrm{SS} 400,14.9 \mathrm{KG} / \mathrm{M}$ & 145,32 & 2,44 \\
\hline & & 2 Angle EQUAL, $50 \mathrm{~W} \times 50 \mathrm{~W} \times 5 \mathrm{~T}, \mathrm{SS} 400,6 \mathrm{M}$ & 387,41 & 6,51 \\
\hline & & 3 Angle EQUAL SIDE-TYPE, 75W $\times 75 \mathrm{~W} \times 9 \mathrm{~T}, 6000 \mathrm{~L}, \mathrm{KSD} 3503, \mathrm{SS} 400,9.96 \mathrm{KG} / \mathrm{M}$ & 169,33 & 2,85 \\
\hline & & 4 Angle EQUAL SIDE-TYPE, 75W $\times 75 \mathrm{~W} \times 6 \mathrm{~T}, 6000 \mathrm{~L}, \mathrm{KSD} 3503, \mathrm{SS} 400,6.85 \mathrm{KG} / \mathrm{M}$ & 194,66 & 3,27 \\
\hline & & 5 Angle EQUAL SIDE-TYPE, $90 \mathrm{~W} \times 90 \mathrm{~W} \times 7 \mathrm{~T}, 6000 \mathrm{~L}, \mathrm{KSD} 3503, \mathrm{SS} 400,9.59 \mathrm{KG} / \mathrm{M}$, & 130,89 & 2,20 \\
\hline \multirow{14}{*}{ Pipe } & \multirow{5}{*}{2016} & 1 PIPE BLACK STEEL, 32Ax6000L,KSD3507,SPP,3.16KG/M & 311,34 & 5,20 \\
\hline & & \begin{tabular}{l|l}
2 PIPE BLACK STEEL,50Ax6000L,KSD3507,SPP,5.12KG/M \\
\end{tabular} & 253,77 & 4,24 \\
\hline & & 3 PIPE BLACK STEEL, 80Ax6000L,KSD3507,SPP,8.49KG/M & 171,16 & 2,86 \\
\hline & & 4 PIPE BLACK STEEL, 25Ax6000L,KSD3507,SPP, 2.45KG/M & 305,12 & 5,09 \\
\hline & & 5 PIPE BLACK STEEL, 150Ax6000L,KSD3507,SPP, 19.2KG/M & 104,99 & $\frac{1,75}{220}$ \\
\hline & \multirow{4}{*}{2017} & 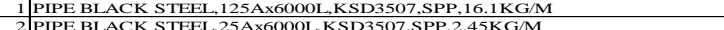 & 144,14 & $\frac{2,20}{5,2}$ \\
\hline & & 3 PIPE BLACK STEEL, $150 \mathrm{~A} \times 6000 \mathrm{~L}, \mathrm{KSD} 3507, \mathrm{SPP}, 19.2 \mathrm{KG} / \mathrm{M}$ & 117,88 & $\frac{5,42}{1,80}$ \\
\hline & & 4 PIPE BLACK STEEL, 65Ax6000L,KSD3507,SPP,6.34KG/M & 210,02 & $\frac{1,00}{3,20}$ \\
\hline & & 5 PIPE BLACK STEEL, 100Ax6000L,KSD3507,SPP, $12.2 \mathrm{KG} / \mathrm{M}$ & 133,03 & 2,03 \\
\hline & \multirow{5}{*}{2018} & 1 PIPE BLACK STEEL, 32Ax6000L,KSD3507,SPP,3.16K G/M & 338,13 & 5,68 \\
\hline & & 2 PIPE BLACK STEEL, 65 Ax6000L,KSD3507,SPP,6.34KG/M & 207,53 & 3,49 \\
\hline & & 3 PIPE BLACK STEEL, 25Ax6000L,KSD3507,SPP,2.45KG/M & 321,54 & 5,41 \\
\hline & & 4 PIPE BLACK STEEL, 100Ax6000L,KSD3507,SPP, $12.2 \mathrm{KG} / \mathrm{M}$ & 116,46 & 1,96 \\
\hline & & 5 PIPE BLACK STEEL, 50Ax6000L,KSD3507,SPP,5.12KG/M & 183,49 & 3,08 \\
\hline \multirow{13}{*}{ H - Beam } & \multirow{4}{*}{2016} & 1 H-Beam $150 \mathrm{H} \times 150 \mathrm{~W} \times 7 \mathrm{~T} \times 10 \mathrm{~T}, \mathrm{KSD} 3503, \mathrm{SS} 400,31.5 \mathrm{KG} / \mathrm{M}, 12 \mathrm{M}$ & 170,10 & 2,84 \\
\hline & & \begin{tabular}{l|l}
2 & H-Beam $125 \mathrm{H} \times 125 \mathrm{~W} \times 6.5 \mathrm{~T} \times 9 \mathrm{~T}, \mathrm{KSD} 3502, \mathrm{SS} 400,23.8 \mathrm{KG} / \mathrm{M}, 12 \mathrm{M}$ \\
\end{tabular} & 176,50 & 2,95 \\
\hline & & \begin{tabular}{l|l}
$\mathrm{H}$-Beam $200 \mathrm{H} \times 200 \mathrm{~W} \times 8 \mathrm{~T} \times 12 \mathrm{~T}, \mathrm{KSD} 3503, \mathrm{SS} 400,49.9 \mathrm{KG} / \mathrm{M}, 12 \mathrm{M}$ \\
\end{tabular} & 121,15 & 2,02 \\
\hline & & 4 H-Beam $100 \mathrm{H} \times 100 \mathrm{~W} \times 6 \mathrm{~T} \times 8 \mathrm{~T}, \mathrm{KSD} 3502, \mathrm{SS} 400,17.2 \mathrm{KG} / \mathrm{M}, 12 \mathrm{M}$ & 173,07 & $\frac{2,89}{2,35}$ \\
\hline & \multirow{4}{*}{2017} & $\begin{array}{l}1 \mathrm{H}-\mathrm{Heam} 200 \mathrm{2} \times 200 \mathrm{~W} \times 8 \mathrm{~T} \times 12 \mathrm{~T}, \mathrm{KSD} 3503, \mathrm{SS} 400,49.9 \mathrm{KG} / \mathrm{M}, 12 \mathrm{M} \\
2\end{array}$ & $\frac{153,82}{155,94}$ & $\frac{2,35}{2,38}$ \\
\hline & & 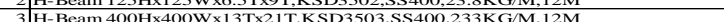 & 155,94 & \\
\hline & & $4 \mathrm{H}$-Beam $250 \mathrm{H} \times 250 \mathrm{~W} \times 9 \mathrm{~T} \times 14 \mathrm{~T}, \mathrm{KSD} 3503, \mathrm{SS} 400,72.4 \mathrm{KG} / \mathrm{M}, 12 \mathrm{M}$ & 02,18 & $\frac{0,95}{1,39}$ \\
\hline & & 5 H-Beam $100 \mathrm{H} \times 100 \mathrm{~W} \times 6 \mathrm{~T} \times 8 \mathrm{~T}, \mathrm{KSD} 3502, \mathrm{SS} 400,17.2 \mathrm{KG} / \mathrm{M}, 12 \mathrm{M}$ & 170,97 & 2,61 \\
\hline & \multirow{5}{*}{2018} & 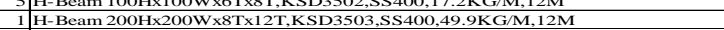 & 148,76 & $\frac{2,61}{2,50}$ \\
\hline & & 2 H-Beam $150 \mathrm{H} \times 150 \mathrm{~W} \times 7 \mathrm{~T} \times 10 \mathrm{~T}, \mathrm{KSD} 3503, \mathrm{SS} 400,31.5 \mathrm{KG} / \mathrm{M}, 12 \mathrm{M}$ & 149,75 & 2,52 \\
\hline & & $3 \mathrm{H}-\mathrm{Beam} 300 \mathrm{H} \times 300 \mathrm{~W} \times 10 \mathrm{~T} \times 15 \mathrm{~T}, \mathrm{KSD} 3503, \mathrm{SS} 400,94 \mathrm{KG} / \mathrm{M}, 12 \mathrm{M}$ & 86,58 & 1,46 \\
\hline & & $4 \mathrm{H}$-Beam $400 \mathrm{H} \times 400 \mathrm{~W} \times 13 \mathrm{~T} \times 21 \mathrm{~T}, \mathrm{KSD} 3503, \mathrm{SS} 400,233 \mathrm{KG} / \mathrm{M}, 12 \mathrm{M}$ & 57,20 & 0,96 \\
\hline & & 5 H-Beam $100 \mathrm{H} \times 100 \mathrm{~W} \times 6 \mathrm{~T} \times 8 \mathrm{~T}, \mathrm{KSD} 3502, \mathrm{SS} 400,17.2 \mathrm{KC}$ & 159,93 & 2,69 \\
\hline \multirow{10}{*}{ Channel } & \multirow{3}{*}{2016} & 1 Channel $100 \mathrm{H} \times 50 \mathrm{~W} \times 5 \mathrm{~T} \times 5 \mathrm{~T}, \mathrm{KSD} 3503, \mathrm{SS} 400,9.36 \mathrm{KG} / \mathrm{M}, 6 \mathrm{M}$ & 350,10 & 5,84 \\
\hline & & 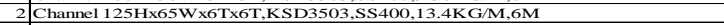 & 243,41 & 4,06 \\
\hline & & \begin{tabular}{c|c|c|} 
Channel $200 \mathrm{H} \times 80 \mathrm{~W} \times 7.5 \mathrm{~T} \times 7.5 \mathrm{~T}, \mathrm{KSD} 3503, \mathrm{SS} 400,24.6 \mathrm{KG} / \mathrm{M}, 6 \mathrm{M}$ \\
\end{tabular} & 113,75 & 1,90 \\
\hline & \multirow{3}{*}{2017} & 1 Channel $80 \mathrm{H} \times 45 \mathrm{~W} \times 3 \mathrm{~T}, \mathrm{SS} 400,6 \mathrm{M} / \mathrm{EA}$ & 301,29 & 4,60 \\
\hline & & 2 Channel $150 \mathrm{OH} \times 75 \mathrm{~W} \times 6.5 \mathrm{~T} \times 6.5 \mathrm{~T}, \mathrm{KSD} 3503, \mathrm{SS} 400,18.6 \mathrm{KG} / \mathrm{M}, 6 \mathrm{M}$ & 169,63 & 2,59 \\
\hline & & $\begin{array}{ll}\text { Channel } 100 \mathrm{H} \times 50 \mathrm{~W} \times 5 \mathrm{~T} \times 5 \mathrm{~T}, \mathrm{KSD} 3503, \mathrm{SS} \\
\end{array}$ & 256,01 & 3,91 \\
\hline & & 1 Channel 100Hx50Wx5Tx5T,KSD3503,SS400,9.36KC & 272,47 & 4,58 \\
\hline & 2018 & \begin{tabular}{l|l}
2 & Channel $200 \mathrm{H} \times 80 \mathrm{~W} \times 7.5 \mathrm{~T} \times 7.5 \mathrm{~T}, \mathrm{KSD} 3503, \mathrm{SS} 400,24.6$ \\
\end{tabular} & 125,79 & 2,11 \\
\hline & & 3 Channel $250 \mathrm{H} \times 90 \mathrm{~W} \times 9 \mathrm{Tx} 9 \mathrm{~T}, \mathrm{KSD} 3503, \mathrm{SS} 400,34.6 \mathrm{KG} / \mathrm{I}$ & 92,23 & 1,55 \\
\hline & & 4 Channel $80 \mathrm{H} \times 45 \mathrm{~W} \times 3 \mathrm{~T}, \mathrm{SS} 400,6 \mathrm{M} / \mathrm{EA}$ & 265,05 & 4,46 \\
\hline & 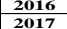 & $\begin{array}{ll}1 & \text { Round Bar 25Dx6000LL,KSD3503,S400,3.85K G/M } \\
1 & \text { Round Bar 19Dx6000,KSD3503,SS400,2.23 KG/M } \\
\end{array}$ & $\frac{26,41}{221,12}$ & $\frac{4,35}{3,37}$ \\
\hline Round Bar & & \begin{tabular}{l|l}
1 & Round Bar 25Dx6000L,KSD3503,SS400,3.85K G/M \\
\end{tabular} & 196,64 & 3,31 \\
\hline & 2018 & \begin{tabular}{l|l}
2 & Round Bar 16Dx6000L,KSD 3503, SS400, $1.58 \mathrm{KG} / \mathrm{M}$ \\
\end{tabular} & 313,01 & 5,26 \\
\hline & 2016 & \begin{tabular}{l|l}
1 & Flat Bar $6000 \mathrm{~L} \times 100 \mathrm{~W} \times 6 \mathrm{~T}, \mathrm{SS} 400,4.71 \mathrm{KG} / \mathrm{M}$ \\
\end{tabular} & 256,94 & 4,29 \\
\hline Flat Bar & 2017 & \begin{tabular}{l|l}
1 & Flat Bar $6000 \mathrm{~L} \times 100 \mathrm{~W} \times 6 \mathrm{~T}, \mathrm{SS} 400,4.71 \mathrm{KG} / \mathrm{M}$ \\
\end{tabular} & 247,82 & 3,78 \\
\hline & 2018 & \begin{tabular}{l|l}
1 & Flat Bar $6000 \mathrm{~L} \times 100 \mathrm{~W} \times 6 \mathrm{~T}, \mathrm{SS} 400,4.71 \mathrm{KG} / \mathrm{M}$ \\
\end{tabular} & 321,26 & 5,40 \\
\hline & 2016 & \begin{tabular}{l|l}
1 & Expanded Metal $50075,33 \mathrm{SW} 75 \mathrm{LW}, 5 \times 7.5,1.2 \times 2.4, \mathrm{LCA}, \mathrm{SS}$
\end{tabular} & 288.88 & 4,82 \\
\hline Expanded Metal & 2017 & 1 Expanded Metal 50075,33SWx75LW,5x7.5,1.2X2.4,LCA,SS & 313,23 & 4,78 \\
\hline & 2018 & 1 Expanded Metal 50075,33SWx75LW,5x7.5,1.2X2.4,LCA,SS400,50, & 204,79 & 3,44 \\
\hline & 2016 & 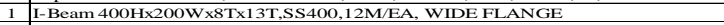 & 36,30 & 0,61 \\
\hline I Beam & 2017 & \begin{tabular}{l|l}
1 & I-Beam 300Hx150W x7Tx9T,SS400,12M, \\
\end{tabular} & 123,31 & 1,88 \\
\hline & & & & \\
\hline
\end{tabular}


Table 4.12 is elaborated to get the optimum ordering point from the calculation results, the order is carried out with qty as listed in table 4.12 and also the frequency of orders made in one year varies from 1 time order in a year to 7 times order in one year.

\section{Calculation of Safety Stock Steel Material Fabrication}

Safety Stock is an additional inventory held to maintain the continuity of production from possible shortages of raw materials. Calculation of standard deviation and safety stock samples for 2016 - 2018 is as follows.

Example Year 2016 angle equal side type $(90 \mathrm{~W} \times 90 \mathrm{~W} \times 7 \mathrm{~T})$.

Table 14. Calculation of Angle deviation of SIDE-TYPE EQUAL, 90Wx90Wx7T

\begin{tabular}{|l|c|c|c|c|}
\hline \multicolumn{1}{|c|}{ Bulan } & Permintaan $X$ & $x$ & $(x-\bar{X})$ & $\left(X_{-}-\bar{X}\right)^{2}$ \\
\hline Januari & 37 & 96,92 & $-59,92$ & 3590,01 \\
\hline Februari & 46 & 96,92 & $-50,92$ & 2592,51 \\
\hline Maret & & 96,92 & $-96,92$ & 9392,84 \\
\hline April & 65 & 96,92 & $-31,92$ & 1018,67 \\
\hline Mei & 277 & 96,92 & 180,08 & 32430,01 \\
\hline Juni & 11 & 96,92 & $-85,92$ & 7381,67 \\
\hline Juli & 313 & 96,92 & 216,08 & 46692,01 \\
\hline Agustus & 17 & 96,92 & $-79,92$ & 6386,67 \\
\hline September & & 96,92 & $-96,92$ & 9392,84 \\
\hline Oktober & 340 & 96,92 & 243,08 & 59089,51 \\
\hline November & 47 & 96,92 & $-49,92$ & 2491,67 \\
\hline Desember & 10 & 96,92 & $-86,92$ & 7554,51 \\
\hline Total & 1163 & & & 188012,92 \\
\hline
\end{tabular}

From table 14, the deviation for 2016 type material angle equal side type (90W x 90W $\mathrm{x} 7 \mathrm{~T}$ ) is $188,012.92$ and the calculation for safety stock is as follows:

$$
\begin{aligned}
& S D=\sqrt{\frac{\sum(X i-\bar{X})^{2}}{n}} \\
& S D=\sqrt{\frac{188012,92}{12}} \\
& S D=\sqrt{15667,74}=125,17 \\
& S S=S D \times Z \\
& S S=125,17 \times 2,33=291,64 \text { unit }
\end{aligned}
$$

\section{Calculation of Reorder Point Steel Material Fabrication}

Reorder Point (ROP) or re-order point is the point of reorder number of raw materials. In this study ROP can be used to find out when companies must place an order, by looking at the inventory of raw materials in the warehouse. By using ROP, companies can find out at what amount of raw materials the company must repurchase so that there is no shortage of raw materials in the warehouse. The components used for the calculation of ROP are annual demand (D), number of working days 264 days, lead time or waiting time from order to goods arriving at the warehouse is 15 days and safety stock.Example calculation for 2016 angle equal side type $(90 \mathrm{~W} \times 90 \mathrm{~W} \times 7 \mathrm{~T})$.

$$
\begin{aligned}
& R O P=(D \times L)+S S \\
& R O P=\left(1163 \times \frac{15}{264}\right)+291,65 \\
& R O P=66,07+291,65=357,73 \text { unit }
\end{aligned}
$$


From the above calculation, the reorder point for 2016 type material angle equal side type ( $90 \mathrm{~W} \times 90 \mathrm{~W} \times 7 \mathrm{~T}$ ) is 357.73 units

\section{Calculation of Total Inventory Costs Company}

The calculation of total inventory costs by company will be calculated using the average inventory available in the company using the following formula:

$$
\text { TIC per }=(\bar{D} x H)+(n x S)
$$

Example calculation for 2016 angle equal side type ( $90 \mathrm{~W} \times 90 \mathrm{~W} \times 7 \mathrm{~T})$.

$$
\begin{aligned}
& \text { TIC per }=(\bar{D} \times H)+(n \times S) \\
& \text { TIC per }=(97 \times 16692,43)+(6 \times 500000) \\
& \text { TIC per }=1617774,4+3000000 \\
& \text { TIC per }=R p 4.617 .774,34
\end{aligned}
$$

From the above calculation, the company's total inventory costs for 2016 type material angle equal side type (90W x $90 \mathrm{~W} \times 7 \mathrm{~T})$ are. $R p 4.617 .774,34$.

\section{Calculation of Total Inventory Costs According to the EOQ Method}

Calculation of total inventory costs according to the EOQ method will be calculated using the EOQ values that have been obtained using the following formula

$$
T I C=\left(\frac{D}{Q} x S\right)+\left(\frac{Q}{2} x H\right)
$$

Example calculation for 2016 angle equal side type $(90 \mathrm{~W} \times 90 \mathrm{~W} \times 7 \mathrm{~T})$.

$$
\begin{aligned}
T I C & =\left(\frac{D}{Q} \times S\right)+\left(\frac{Q}{2} \times H\right) \\
T I C * & =\left(\frac{1163}{263,96} \times 500000\right)+\left(\frac{263,96}{2} \times 16692,43\right) \\
T I C * & =2203025,9+2203026 \\
T I C * & =R p 4.406 .051,76
\end{aligned}
$$

From the above calculation, the total inventory cost of the EOQ method for 2016 type of material angle equal side type ( $90 \mathrm{~W} \times 90 \mathrm{~W} \times 7 \mathrm{~T})$ is $R p 4.406 .051,76$.

\section{Comparison of Research Results with Company Policy}

Comparison of the total cost of raw material inventory according to EOQ with the total raw material inventory run by the company in 2016-2018 can be seen in the table below:

Table 15. TIC According to EOQ and TIC Run by the Company and Savings During the 2016-2018 Period

\begin{tabular}{|c|cr|rr|rr|c|}
\hline \multirow{2}{*}{ Tahun } & \multirow{2}{*}{ TIC Metode Perusahaan } & \multicolumn{2}{|c|}{ TIC Metode EOQ } & \multicolumn{3}{|c|}{ Penghematan } \\
\cline { 5 - 8 } & & & & & \multicolumn{2}{|c|}{ Rupiah } & Persentase \\
\hline $\mathbf{2 0 1 6}$ & $\mathrm{Rp}$ & $77.932 .612,06$ & $\mathrm{Rp}$ & $69.371 .506,24$ & $\mathrm{Rp}$ & $8.561 .105,82$ & $10,98 \%$ \\
\hline $\mathbf{2 0 1 7}$ & $\mathrm{Rp}$ & $81.533 .693,58$ & $\mathrm{Rp}$ & $69.319 .824,16$ & $\mathrm{Rp}$ & $12.213 .869,42$ & $14,98 \%$ \\
\hline $\mathbf{2 0 1 8}$ & $\mathrm{Rp}$ & $88.861 .779,13$ & $\mathrm{Rp}$ & $78.159 .819,71$ & $\mathrm{Rp}$ & $10.701 .959,41$ & $12,04 \%$ \\
\hline
\end{tabular}

Based on Table 15 generated total inventory cost savings of Rp. 8,561,105.82 in 2016, Rp. 12,213,869.42 in 2017 and Rp. 10,701,959.41 in 2018. If the savings made are in percent, the savings in 2016 will be $10.98 \%, 2017$ will be $14.98 \%$ and 2018 will be $12.04 \%$. Comparison of the results obtained from this study with the policies adopted by the company can be seen in the table below. 
Table 16. Comparison of Q Value of EOQ method with Company Q Value, Research Reorder Point and Company Reorder Point, Safety Research Stock and Company Safety Stock in 2016 - 2018.

\begin{tabular}{|c|c|c|c|c|c|c|c|c|c|c|c|}
\hline \multirow[b]{2}{*}{ Kategori } & \multirow[b]{2}{*}{ Tahum } & \multirow[b]{2}{*}{ No } & \multirow[b]{2}{*}{ Deskripsi } & \multicolumn{4}{|c|}{ Perbandingan Metode EOQ Dengan Metode Perusahaan } & \multicolumn{2}{|c|}{ Reorder Point (Unit) } & \multicolumn{2}{|c|}{ Safety Stock (Unit) } \\
\hline & & & & $\begin{array}{c}\text { Metode EOQ } \\
\text { (Unit) }\end{array}$ & $\begin{array}{c}\text { Frekuensi Pemesanan } \\
\text { (EOQ) }\end{array}$ & $\begin{array}{c}\text { Metode Q } \\
\text { Perusahaan } \\
\text { (Unit) }\end{array}$ & \begin{tabular}{|c|}
$\begin{array}{c}\text { Frekuensi Pemesanan } \\
\text { (Perusahaaan) }\end{array}$ \\
\end{tabular} & Penelitian & Perusahaan & Penelitian & Perusahaan \\
\hline \multirow{14}{*}{ Angle } & \multirow{4}{*}{2016} & & Angle EQUAL SIDE-TYPE, $90 \mathrm{~W} \times 90 \mathrm{~W} \times 7 \mathrm{~T}$ & 263.96 & 4 & 193.83 & 6 & 358 & 194 & 292 & - \\
\hline & & \begin{tabular}{|l|l}
2 &
\end{tabular} & Angle EQUAL SIDE-TYPE,75Wx75Wx9T & 211.97 & 4 & 125.00 & 6 & 193 & 125 & 150 & - \\
\hline & & \begin{tabular}{|l|}
3 \\
\end{tabular} & Angle EQUAL SIDE-TYPE,100Wx100Wx10T & 145.01 & 2 & 58.50 & 6 & 97 & 59 & 77 & - \\
\hline & & 4 & Angle EQUAL SIDE-TYPE, $75 \mathrm{~W} \times 75 \mathrm{~W} \times 6 \mathrm{~T}$ & 197.94 & 3 & 109.00 & 6 & 171 & 109 & 134 & - \\
\hline & & 12 & Angle EQUAL SIDE-TYPE, $90 \mathrm{~W} \times 90 \mathrm{~W} \times 7 \mathrm{~T}$ & 295.14 & 5 & 221.50 & 6 & 337 & 222 & 261 & - \\
\hline & & \begin{tabular}{|l|ll}
2 & $t$
\end{tabular} & Angle EQUAL SIDE-TYPE, $75 \mathrm{~W} \times 75 \mathrm{~W} \times 9 \mathrm{~T}$ & 256.27 & 4 & 167.00 & 6 & 251 & 167 & 194 & - \\
\hline & 2017 & 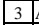 & Angle EQUAL SIDE-TYPE,100Wx100Wx10T & 173.64 & 3 & 76.67 & 6 & 139 & 77 & 112 & - \\
\hline & & 45 & Angle EQUAL SIDE-TYPE,65W x65Wx6T & 348.96 & 5 & 309.67 & 6 & 497 & 310 & 391 & - \\
\hline & & 5 & Angle EQUAL SIDE-TYPE,75W x75W x6T & 242.60 & 4 & 149.67 & 6 & 263 & 150 & 212 & - \\
\hline & & 1 & Angle EQUAL SIDE-TYPE, $100 \mathrm{~W} \times 100 \mathrm{~W} \times 10 \mathrm{~T}$ & 145.32 & 2 & 59.17 & 6 & 139 & 59 & 119 & - \\
\hline & & 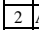 & Angle EQUAL,50Wx50Wx5T & 387.41 & 7 & 420.50 & 6 & 583 & 421 & 440 & - \\
\hline & 2018 & 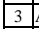 & Angle EQUAL SIDE-TYPE,75Wx75Wx9T & 169.33 & 3 & 80.33 & 6 & 184 & 80 & 156 & - \\
\hline & & \begin{tabular}{|l|l}
4 & 1
\end{tabular} & Angle EQUAL SIDE-TYPE,75Wx75Wx6T & 194.66 & 3 & 106.17 & 6 & 131 & 106 & 95 & - \\
\hline & & 5 & Angle EQUAL SIDE-TYPE,90Wx90Wx7T & 130.89 & 2 & 72.00 & 4 & 110 & 72 & 94 & - \\
\hline & & & PIPE BLACK STEEL,32Ax6000L,KSD3507,SPP,3.16KG/M & 311.34 & 5 & 269.67 & 6 & 438 & 270 & 346 & - \\
\hline & & \begin{tabular}{|l|ll}
2 & $\mathrm{I}$
\end{tabular} & PIPE BLACK STEEL,50Ax6000L,KSD3507,SPP,5.12KG/M & 253.77 & 4 & 179.17 & 6 & 239 & 179 & 177 & - \\
\hline & 2016 & \begin{tabular}{|l|lll}
3 & $\mathrm{I}$ \\
\end{tabular} & PIPE BLACK STEEL,80Ax6000L,KSD3507,SPP,8.49KG/M & 171.16 & 3 & 81.50 & 6 & 125 & 82 & 97 & - \\
\hline & & \begin{tabular}{|l|l|l}
4 & 1 \\
\end{tabular} & PIPE BLACK STEEL, 25Ax6000L,KSD3507,SPP,2.45KG/M & 305.12 & 5 & 259.00 & 6 & 389 & 259 & 301 & - \\
\hline & & $5 \mathrm{i}$ & PIPE BLACK STEEL,150Ax6000L,KSD3507,SPP,19.2KG/M & 104.99 & 2 & 46.00 & 4 & 58 & 46 & 48 & - \\
\hline & & $1 \mathrm{I}$ & PIPE BLACK STEEL,125Ax6000L,KSD3507,SPP,16.1KG/M & 144.14 & 2 & 79.25 & 4 & 88 & 79 & 70 & - \\
\hline & & \begin{tabular}{|l|lll}
2 & 1
\end{tabular} & PIPE BLACK STEEL,25Ax6000L,KSD3507,SPP,2.45KG/M & 355.11 & 5 & 320.67 & 6 & 414 & 321 & 305 & - \\
\hline Pipe & 2017 & 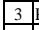 & PIPE BLACK STEEL, 150Ax6000L,KSD3507,SPP,19.2KG/M & 117.88 & 2 & 53.00 & 4 & 62 & 53 & 50 & - \\
\hline & & 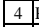 & PIPE BLACK STEEL,65Ax6000L,KSD3507,SPP,6.34KG/M & 210.02 & 3 & 112.17 & 6 & 193 & 112 & 155 & - \\
\hline & & 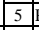 & PIPE BLACK STEEL,100Ax6000L,KSD3507,SPP,12.2KG/M & 133.03 & 2 & 67.50 & 4 & 94 & 68 & 78 & - \\
\hline & & $1 \mathrm{i}$ & PIPE BLACK STEEL,32Ax6000L,KSD3507,SPP,3.16KG/M & 338.13 & 6 & 320.33 & 6 & 452 & 320 & 343 & - \\
\hline & & \begin{tabular}{|l|lllll}
2 & $\mathrm{I}$
\end{tabular} & PIPE BLACK STEEL,65Ax6000L,KSD3507,SPP,6.34KG/M & 207.53 & 3 & 120.67 & 6 & 211 & 121 & 169 & - \\
\hline & 2018 & \begin{tabular}{|l|llll}
3 & $\mathrm{I}$ \\
\end{tabular} & PIPE BLACK STEEL,25Ax6000L,KSD3507,SPP,2.45KG/M & 321.54 & 5 & 289.67 & 6 & 331 & 290 & 233 & - \\
\hline & & $4 \mathrm{H}$ & PIPE BLACK STEEL,100Ax6000L,KSD3507,SPP,12.2KG/M & 116.46 & 2 & 57.00 & 4 & 60 & 57 & 47 & - \\
\hline & & 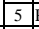 & PIPE BLACK STEEL,50Ax6000L,KSD3507,SPP,5.12KG/M & 183.49 & 3 & 94.33 & 6 & 124 & 94 & 92 & - \\
\hline & & & H-Beam 150Hx150Wx7Tx10T,KSD3503,SS400,31.5KG/M,12M & 170.10 & 3 & 80.50 & 6 & 125 & 81 & 98 & - \\
\hline & & $21 \mathrm{I}$ & H-Beam 125Hx125Wx6.5Tx9T,KSD3502,SS400,23.8KG/M,12M & 176.50 & 3 & 86.67 & 6 & 165 & 87 & 135 & - \\
\hline & 2016 & & H-Beam 200Hx200Wx8Tx12T,KSD3503,SS400,49.9KG/M,12M & 121.15 & 2 & 61.25 & 4 & 69 & 61 & 55 & - \\
\hline & & \begin{tabular}{|l|l}
4 & 1
\end{tabular} & H-Beam 100Hx100Wx6Tx8T,KSD3502,SS400,17.2KG/M,12M & 173.07 & 3 & 83.33 & 6 & 187 & 83 & 159 & - \\
\hline & & & H-Beam 200Hx200Wx8Tx12T,KSD3503,SS400,49.9KG/M,12M & 153.82 & 2 & 60.17 & 6 & 99 & 60 & 79 & - \\
\hline & & $21 \mathrm{l}$ & H-Beam 125Hx125Wx6.5Tx9T,KSD3502,SS400,23.8KG/M,12M & 155.94 & 2 & 61.83 & 6 & 118 & 62 & 97 & - \\
\hline & 2017 & \begin{tabular}{|l|llll}
3 & 1
\end{tabular} & H-Beam 400H×400Wx13Tx21T,KSD3503,SS400,233KG/M,12M & 62.18 & 1 & 19.67 & 3 & 18 & 20 & 15 & - \\
\hline H-Beam & & \begin{tabular}{|l|llll}
4 & 1
\end{tabular} & H-Beam 250Hx250Wx9Tx14T,KSD3503,SS400,72.4KG/M,12M & 91.23 & 1 & 31.75 & 4 & 39 & 32 & 32 & - \\
\hline & & $5 \mathrm{i}$ & H-Beam 100Hx100Wx6Tx8T,KSD3502,SS400,17.2KG/M,12M & 170.97 & 3 & 74.33 & 6 & 95 & 74 & 69 & - \\
\hline & & & H-Beam 200Hx200Wx8Tx12T,KSD3503,SS400,49.9KG/M,12M & 148.76 & 3 & 62.00 & 6 & 54 & 62 & 32 & - \\
\hline & & \begin{tabular}{|l|llll}
2 & 1
\end{tabular} & H-Beam 150Hx150Wx7Tx10T,KSD3503,SS400,31.5KG/M,12M & 149.75 & 3 & 62.83 & 6 & 77 & 63 & 56 & - \\
\hline & 2018 & & H-Beam 300Hx300Wx10Tx15T,KSD3503,SS400,94KG/M,12M & 86.58 & 1 & 31.50 & 4 & 28 & 32 & 21 & - \\
\hline & & \begin{tabular}{|l|l}
4 & 1 \\
\end{tabular} & H-Beam 400H×400Wx13T×21T,KSD3503,SS400,233KG/M,12M & 57.20 & 1 & 27.50 & 2 & 20 & 28 & 17 & - \\
\hline & & 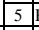 & H-Beam 100Hx100Wx6Tx8T,KSD3502,SS400,17.2KG/M,12M & 159.93 & 3 & 71.67 & 6 & 75 & 72 & 51 & - \\
\hline & & & Channel 100Hx50Wx5Tx5T,KSD3503,SS400,9.36KG/M,6M & 350.10 & 6 & 341.00 & 6 & 543 & 341 & 426 & - \\
\hline & 2016 & 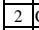 & Channel 125Hx65Wx6Tx6T,KSD3503,SS400,13.4KG/M,6M & 243.41 & 4 & 164.83 & 6 & 217 & 165 & 160 & - \\
\hline & & & Channel 200Hx80Wx7.5Tx7.5T,KSD3503,SS400,24.6KG/M,6M & 113.75 & 2 & 54.00 & 4 & 69 & 54 & 56 & - \\
\hline & & 18 & Channel $80 \mathrm{H} \times 45 \mathrm{~W} \times 3 \mathrm{~T}, \mathrm{SS} 400,6 \mathrm{M} / \mathrm{EA}$ & 301.29 & 5 & 230.83 & 6 & 311 & 231 & 232 & - \\
\hline & 2017 & & Channel 150Hx75Wx6.5Tx6.5T,KSD3503,SS400,18.6KG/M,6M & 169.63 & 3 & 73.17 & 6 & 121 & 73 & 96 & - \\
\hline Channel & & \begin{tabular}{|c|cccc}
3 & 6
\end{tabular} & Channel 100Hx50Wx5Tx5T,KSD3503,SS400,9.36KG/M,6M & 256.01 & 4 & 166.67 & 6 & 269 & 167 & 213 & - \\
\hline & & 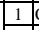 & Channel 100H×50Wx5Tx5T,KSD3503,SS400,9.36KG/M,6M & 272.47 & 5 & 208.00 & 6 & 266 & 208 & 196 & - \\
\hline & & & Channel $200 \mathrm{H} \times 80 \mathrm{~W} \times 7.5 \mathrm{~T} \times 7.5 \mathrm{~T}, \mathrm{KSD} 3503, \mathrm{SS} 400,24.6 \mathrm{KG} / \mathrm{M}, 6 \mathrm{M}$ & 125.79 & 2 & 66.50 & 4 & 80 & 67 & 65 & - \\
\hline & 2018 & & Channel 250Hx90Wx9Tx9T,KSD3503,SS400,34.6KG/M,6M & 92.23 & 2 & 47.67 & 3 & 49 & 48 & 40 & - \\
\hline & & & Channel $80 \mathrm{H} \times 45 \mathrm{~W} \times 3 \mathrm{~T}, \mathrm{SS} 400,6 \mathrm{M} / \mathrm{EA}$ & 265.05 & 4 & 196.83 & 6 & 171 & 197 & 104 & - \\
\hline & 2016 & $1 \mathrm{H}$ & Round Bar 25Dx6000L,KSD3503,SS400,3.85KG/M & 260.41 & 4 & 188.67 & 6 & 333 & 189 & 268 & - \\
\hline & 2017 & $1 \mathrm{H}$ & Round Bar 19Dx6000L,KSD3503,SS400,2.23KG/M & 221.12 & 3 & 124.33 & 6 & 214 & 124 & 171 & - \\
\hline Round Bar & & & Round Bar 25Dx6000L,KSD3503,SS400,3.85KG/M & 196.64 & 3 & 108.33 & 6 & 213 & 108 & 176 & - \\
\hline & 2018 & & Round Bar 16Dx6000L,KSD3503,SS400,1.58KG/M & 313.01 & 5 & 274.50 & 6 & 260 & 275 & 167 & - \\
\hline & 2016 & & Flat Bar $6000 \mathrm{Lx} 100 \mathrm{~W} \times 6 \mathrm{~T}, \mathrm{SS} 400,4.71 \mathrm{KG} / \mathrm{M}$ & 256.94 & 4 & 183.67 & 6 & 262 & 184 & 199 & - \\
\hline Flat Bar & 2017 & $1 \mathrm{I}$ & Flat Bar $6000 \mathrm{Lx} 100 \mathrm{~W} \times 6 \mathrm{~T}, \mathrm{SS} 400,4.71 \mathrm{KG} / \mathrm{M}$ & 247.82 & 4 & 156.17 & 6 & 165 & 156 & 111 & - \\
\hline & 2018 & $1 \mathrm{I}$ & Flat Bar 6000Lx100Wx6T,SS400,4.71KG/M & 321.26 & 5 & 289.17 & 6 & 281 & 289 & 183 & - \\
\hline & 2016 & $1 \mathrm{H}$ & Expanded Metal 50075,33SWx75LW,5x7.5,1.2X2.4,LCA,SS400,50, & 288.88 & 5 & 232.17 & 6 & 309 & 232 & 230 & - \\
\hline $\begin{array}{l}\text { Expanded } \\
\text { Metal }\end{array}$ & 2017 & & Expanded Metal 50075,33SWx75LW,5x7.5,1.2X2.4,LCA,SS400,50, & 313.23 & 5 & 249.50 & 6 & 310 & 250 & 225 & - \\
\hline & 2018 & $1 \mathrm{I}$ & Expanded Metal 50075,33SWx75LW,5x7.5,1.2X2.4,LCA,SS400,50, & 204.79 & 3 & 117.50 & 6 & 117 & 118 & 77 & - \\
\hline & 2016 & 11 & I-Beam 400Hx200W x8Tx13T,SS400,12M/EA, WIDE FLANGE & 36.30 & 1 & 11.00 & 2 & 8 & 11 & 7 & - \\
\hline I Beam & 2017 & 11 & I-Beam 300Hx150Wx7Tx9T,SS400,12M, WIDE FLANGE & 123.31 & 2 & 38.67 & 6 & 70 & 39 & 57 & - \\
\hline & 2018 & & I-Beam 400H×200Wx8Tx13T,SS400,12M/EA, WIDE FLANGE & 61.22 & & 21.00 & 3 & 17 & 21 & 13 & \\
\hline
\end{tabular}

Based on Table 16 the results obtained $Q$ value (the number of messages each time) based on the results of research and methods that have been applied in the company. The frequency of orders made by the company when seen from the $\mathrm{Q}$ value of the company ranges from 6 orders per year for each type of material means that on average every 2-3 months the company orders it. While the results of ordering frequency based on the EOQ method vary according to the level of need and usage, the order frequency varies greatly from 1 time to 6 times a year. This EOQ method is one of the ways to control inventory which is 
part of the fields included in the SCOR (Supply Chain Operation Reference) model because it includes interactions in the company's supply chain that explains the mapping done to get a clear model picture of the material flow of information flow, financial flow, from a company's supply chain. This is in line with the results of Ahmad Hidayat Sutawijaya's research (MIX: Journal of Management Scientific, Volume VI, No. 1, February 2016).

Based on Table 16, the results of the company's ROP value tend to be smaller than the ROP results of the EOQ method and the average ROP value of the company is smaller than the EOQ method because the company still conducts ROP only based on estimates and applies an average order every two months meaning ordering only done when the stock in the field is running low.

Based on Table 16 we can see that the company has not yet owned and implemented a safety stock system, so a state of stock out occurs when the plant needs certain material. Then based on the EOQ method a safety stock is calculated and the results vary according to the annual needs of each material.

\section{CONCLUSION}

Based on the analysis and calculation results that have been obtained, it can be concluded that a comparative analysis of the effectiveness of inventory control using the EOQ (economic order quantity) method with the company method on steel material fabrication Krakatau Posco that the steel material fabrication inventory control method with the EOQ method is proven to be more effective, efficient and better than the company's manual method. Starting from the results of the EOQ value, ROP, safety stock and from the calculation results of Krakatau Posco's total inventory cost can save inventory costs by Rp 8,561,105 (10.98\%), - in 2016, Rp 12,213,869 (14.98\%), - in 2017 and IDR 10,701,959 $(12.04 \%)$

\section{REFERENCE}

Agus Harjito and Martono. 2011. Management Finance. Second Edition, First Matter, Yogyakarta: EKONISIA Publisher.

Agus Ristono. 2013. Inventory Management. Graha Ilmu Publisher,Yogyakarta.

Agarwal Sachin, 2014, Economic Order Quantity Model : A Review VSRD International

Journal of Mechanical, Civil, Automobile and Production Engineering, Vol. IV Issue XII

December 2014.

Alexandri, Moh. Benny. 2009. Business Financial Management: Theory and Questions. Bandung: Alfabeta Publisher.

Andiana Mellisa, 2018, Application of EOQ Method in Controlling Raw Material Inventory of PT X. Maranatha Accounting Journal Volume 10 Number 1, May 2018: 30-40.

Andreano, Langke, Merlyn M., 2018, Analysis of Inventory Control of Coconut Raw

Materials at PT. Tropica Cocoprima Using Economic Order Quantity. EMBA Journal Vol.6 No.3 July 2018, Pg. 1158 - 1167.

Anik, and Abdul, 2018, Analysis of Raw Material Inventory Control Using Methods EOQ di PT. X Management System \& Industrial Engineering journal Vol 1 No.2.

Apriyani Noor .2017. Analysis of Raw Material Inventory Control Using Economic Order Quantity and Kanban Methods at PT. Adyawinsa Stamping Industries. Journal of Option OPTIONS Vol 10 No 2

Assauri, Sofyan. 2011. Production and Operations Management. Jakarta: CP- FEUI.

Darsono, 2017, Inventory Control System with EOQ Method Using Genetic Algorithms

(Gundaling Farm Case Study). Journal of ISD Vol.2 No.1 January - June 2017. 
Dwi Putra and Agoes, 2019, Analysis of Inventory Management Performance in Barjaz Company Using the EOQ Approach. Eud Management E-Journal, Vol. 8, No. 1, 2019: 7163 - 7190 .

Elwidho, Achmad, 2016, Analysis of Raw Material Inventory Control Using the Economic Order Quantity Method in Bonansa Bread Company. Management Analysis Journal 5 (4) (2016).

Gede Agus, Darmawan, Wayan Cipta, \& Ni Nyoman, 2015, Application of Economic Order Quantity (EOQ) in the management of flour raw material supplies in the PIA ARIAWAN business in Banyuwangi Village. e-Journal Bisma Ganesha Educational University Department of Management (Volume 3 2015).

Guga,and, Orjola, 2015, Inventory Management through EOQ model aCase Study of Shpresa LTD, Albania. International Journal of Economics, Commerce and Management United Kingdom Vol. III, Issue 12, December 2015. Handoko, Hani T. 2011. Fundamentals of Production and Operations Management. Surakarta:

BPFE.

Heizer, Jay dan Barry Render. 2010. Operation management. Ninth Edition. Jakarta: Salemba Empat.

Hasian D.P. 2016, The concept of Minimum Inventory Maximum Control Part of PT. Semen Padang mining heavy equipment. Journal of Industrial Systems Optimization, Vol. 11 No. 1, April 2012: 203-207.

Indonesian Accounting Association. 2014. Introduction to Financial Accounting. Palembang: Indonesian Accounting Association of South Sumatra Region. Paduloh, Rio, 2018, Analysis of Inventory Control of Iron Plate Body Caroseri Industry Raw Materials Using the EOQ Method (Case Study at PT.MISITAMA). Journal of Industrial Manufacturing Vol. 3, No. 1, January 2018, pp. 37-44.

Prawirosentono, Suyadi. 2009. Operations Management: Analysis and Case Study. Fourth Edition. Jakarta: Bumi Aksara.

Pujawan, I., N., dan Mahendrawathi. 2017. Supply Chain Management, Third Edition, Guna Widya, Surabaya.

Rakes.k. 2016, Economic Order Quantity (EOQ) Model .Global Journal of Finance and Economic Management. Volume 5, Number 1 (2016), pp. 1-5.

Schroeder, Goldstein and Rungtusanatham. 2010. Operations Management: Contemporary Concepts and Cases. 5th ed.. McGraw-Hill.

Sofyan, Diana Khairani. 2013. Production Planning and Control. Lhokseumawe NAD: Penerbit Graha Ilmu.

Sugiyono. 2011. Administrative Research Methods. Bandung: Alfabeta.

Sutawijaya \& Marlapa.2016. Supply Chain Management Analisa dan Penerapan Mengunakan Reference (SCOR) di PT. Indoturbine. MIX: Jurnal Ilmiah Manajemen, Volume VI, No. 1. 121-138.

Taufiq, Daniel, Muhammad, 2017, Inventory Based Inventory Management Application Economic Order Quantity (EOQ), . Jurnal JTIK, 1(1) 2017, 48-60.

Tungalag, Erdenebat, 2017. A Note on Economic Order Quantity Model). iBusiness, 2017, 9, 74-79.

Yamit, Z. (2011), “Inventory Management ", Ekonisia, Campus of the Faculty of Economics UII,

Yogyakarta. 